Article

\title{
Spin-ARPES EUV Beamline for Ultrafast Materials Research and Development
}

\author{
Zhonghui Nie ${ }^{1,+}$, Ion Cristian Edmond Turcu 1,2,*, Yao Li ${ }^{1,+}$, Xiaoqian Zhang 1, ${ }^{+}$, Liang He ${ }^{1, *}$, \\ Jian Tu ${ }^{1}$, Zhiqiang $\mathrm{Ni}^{1}$, Huangfeng $\mathrm{Xu}{ }^{1}$, Yequan Chen ${ }^{1}$, Xuezhong Ruan ${ }^{1}$, Fabio Frassetto ${ }^{3,+} \mathbb{D}$, \\ Paolo Miotti ${ }^{3,+}$, Nicola Fabris ${ }^{3,+}$, Luca Poletto ${ }^{3,+}$, Jing Wu ${ }^{4} \mathbb{D}$, Qiangsheng Lu ${ }^{5}$, Chang Liu ${ }^{5}$, \\ Thorsten Kampen ${ }^{6}$, Ya Zhai ${ }^{7}$, Wenqing Liu ${ }^{8}$ (D), Cephise Cacho ${ }^{9}$, Xuefeng Wang ${ }^{1}$ (D), \\ Fengqiu Wang ${ }^{1}$, Yi Shi ${ }^{1}$, Rong Zhang ${ }^{1}$ and Yongbing $\mathrm{Xu}^{1,10 \text {,* }}$ \\ 1 School of Electronic Science and Engineering, Nanjing University, Nanjing 210023, China; \\ niezhonghui1991@163.com (Zho.N.); liyao@nju.edu.cn (Y.L.); xiaoqianqian_zhang@163.com (X.Z.); \\ 15651631979@163.com (J.T.); 13739194032@163.com (Zhi.N.); 15251897129@163.com (H.X.); \\ chenyequan163@163.com (Y.C.); xzruan@nju.edu.cn (X.R.); xfwang@nju.edu.cn (X.W.); \\ fwang@nju.edu.cn (F.W.); yshi@nju.edu.cn (Y.S.); rzhang@nju.edu.cn (R.Z.) \\ 2 STFC Rutherford Appleton Laboratory, Central Laser Facility, Oxfordshire OX11 0QX, UK \\ 3 National Research Council Institute of Photonics and Nanotechnologies, 35131 Padova, Italy; \\ fabio.frassetto@cnr.it (F.F.); paolo.miotti@pd.ifn.cnr.it (P.M.); fabris.nicola@pd.ifn.cnr.it (N.F.); \\ luca.poletto@cnr.it (L.P.) \\ 4 Department of Physics, York University, York YO10 5DD, UK; jing.wu@york.ac.uk \\ 5 Shenzhen Institute for Quantum Science and Engineering, and Department of Physics, Southern University \\ of Science and Technology, Shenzhen 518055, China; q16zx@mail.missouri.edu (Q.L.); \\ liuc@sustc.edu.cn (C.L.) \\ 6 Department of Solid State Physics, Technische Universität Berlin, 10623 Berlin, Germany; \\ thorsten.kampen@specs.com \\ 7 Department of Physics, Southeast University, Nanjing 210009, China; yazhai@seu.edu.cn \\ 8 Department of Electronic Engineering, Royal Holloway University of London, Egham TW20 0EX, UK; \\ wenqing.liu@rhul.ac.uk \\ 9 Diamond Light Source Ltd., Harwell Campus, Oxfordshire OX11 0QX, UK; cephise.cacho@diamond.ac.uk \\ 10 York-Nanjing Joint Center in Spintronics, Department of Electronic Engineering, The University of York, \\ York YO10 5DD, UK \\ * Correspondence: edmondturcu@nju.edu.cn or edmond.turcu@stfc.ac.uk (I.C.E.T.); \\ heliang@nju.edu.cn (L.H.); ybxu@nju.edu.cn (Y.X.) \\ + These authors contribute equally to this work.
}

Received: 12 December 2018; Accepted: 15 January 2019; Published: 22 January 2019

\begin{abstract}
A new femtosecond, Extreme Ultraviolet (EUV), Time Resolved Spin-Angle Resolved Photo-Emission Spectroscopy (TR-Spin-ARPES) beamline was developed for ultrafast materials research and development. This 50-fs laser-driven, table-top beamline is an integral part of the "Ultrafast Spintronic Materials Facility", dedicated to engineering ultrafast materials. This facility provides a fast and in-situ analysis and development of new materials. The EUV source based on high harmonic generation process emits $2.3 \times 10^{11}$ photons/second $\left(2.3 \times 10^{8}\right.$ photons/pulse) at $\mathrm{H} 23$ $(35.7 \mathrm{eV})$ and its photon energy ranges from $10 \mathrm{eV}$ to $75 \mathrm{eV}$, which enables surface sensitive studies of the electronic structure dynamics. The EUV monochromator provides the narrow bandwidth of the EUV beamline while preserving its pulse duration in an energy range of 10-100 eV. Ultrafast surface photovoltaic effect with $\sim 650$ fs rise-time was observed in p-GaAs (100) from time-resolved ARPES spectra. The data acquisition time could be reduced by over two orders of magnitude by scaling the laser driver from $1 \mathrm{KHz}, 4 \mathrm{~W}$ to $\mathrm{MHz}, \mathrm{KW}$ average power.
\end{abstract}

Keywords: high-order harmonic generation; ultrafast extreme violet; time-resolved ARPES 


\section{Introduction}

Angular Resolved PhotoElectron Spectroscopy (ARPES) is one of the most powerful techniques for the investigation of the electronic energy levels and momenta (k-vectors) at the surface of a solid material, resulting in detailed characterization of the energy band dispersion and Fermi surface [1]. The Extreme Ultraviolet (EUV) radiation from Synchrotron Radiation Sources (SRS) provides the narrow-bandwidth radiation to measure electron energy levels with excellent resolutions of $<10 \mathrm{meV}$.

The SRS-driven beamline was miniaturized to table-top laser-driven laboratory-size ARPES using UV photons $(\sim 6 \mathrm{eV})$ for remarkable researches in superconducting materials [2,3]. This was possible by the advent of chirp pulse amplification (CPA) technique [4], generating high-power femtosecond laser pulses [2]. The laser-driven ARPES was then extended to $\sim 7 \mathrm{eV}$ UV photons [5] and $\sim 10.5 \mathrm{eV}$ in order to increase the electronic momentum space [6-8]. New Vacuum Ultra-Violet (VUV) sources which use metasurfaces to convert TiS laser pulses to $6 \mathrm{eV}$ [9] and $4.5 \mathrm{eV}$ radiation [10] have been reported but have not yet been used for ARPES.

The laser photoelectron spectroscopy can be extended to the time-domain taking advantage of the femtosecond pulse duration of the laser-driver [11] and opening the field of time-resolved ARPES (TR-ARPES) [12-16]. In this technique, the femtosecond laser pulse is split into two pulses with a beam-splitter. The first pulse ( $\mathrm{h} v_{\text {excitation }} \sim 1.5 \mathrm{eV}$ in case of Ti: Sapphire laser systems) excites electrons in the sample material. The second pulse is converted-through nonlinear crystals-to UV (typically $\mathrm{h} v_{\text {probe }} \sim 6 \mathrm{eV}$ ) and probes the sample material by extracting photoelectrons (PE) which are then analyzed in the ARPES. By delaying the UV probe pulse with respect to the Infrared (IR) excitation pulse, one obtains a time sequence of ARPES spectra, which constitute the "video" of the electron-motion inside the material.

The femtosecond-laser-based TR-ARPES technique was further developed by extending the probe photon energy from UV range to EUV range in order to access the full Brillouin zone of the sample material. This was achieved by converting the laser photons to EUV photons through the High Harmonic Generation (HHG) process [17-22]. For example, in the Artemis facility [23-25], an excitation pulse generated by an optical parametric amplifier (OPA), tunable from the near-infrared (NIR) to mid-IR (photon energy from $1 \mathrm{eV}$ to $60 \mathrm{meV}$ ), works as pump pulse and a femtosecond HHG-based EUV pulse acts as probe pulse, whose photon energy ranges from $10 \mathrm{eV}-100 \mathrm{eV}$ by means of a pulse-preserving monochromator [26,27]. This new type of facility, incorporating HHG EUV pulses with $\sim 20 \mathrm{fs}$ duration ( $\sim 10 \mathrm{fs}$ optional) and photon energies extending to $\sim 100 \mathrm{eV}$, led to the discovery of several ultra-fast processes in novel materials, such as $2 \mathrm{D}$ materials like Graphene, $\mathrm{TaS}_{2}$, and topological insulators [28-30]. Several laboratories around the world have developed such EUV beamlines for time-domain research in both materials science and atomic-molecular physics [31-39].

In this paper, we present the new femtosecond EUV beamline for time-resolved Spin-ARPES, developed for research on ultrafast and spintronic materials. The laser-driven, sec-top beamline is an integral part of the "Ultrafast Spintronic Materials Facility" at Nanjing University. This facility stations are dedicated to materials engineering by molecular beam epitaxy (MBE), sputtering and pulsed laser deposition (PLD). The sample material is easily transferred from the materials engineering stations to the TR-Spin-ARPES end-station through a fast and contamination-free ultrahigh vacuum (UHV) transport tube. The facility can provide the in-situ analysis for novel materials, especially for those sensitive materials, in order to reveal their intrinsic properties.

The paper describes the beamline components shown schematically in Figures 1 and 2: TiS laser-driver with $\sim 50 \mathrm{fs}$ pulse duration, $4 \mathrm{~W}$ average power and $1 \mathrm{kHz}$ repetition rate (Section 2); HHG EUV Source generating photons with energies $>75 \mathrm{eV}$, with maximum flux of single harmonic $>10^{11}$ photons/second $(\mathrm{ph} / \mathrm{s}), \sim 50$ fs pulse duration ( $>10^{8} \mathrm{ph} / \mathrm{pulse} /$ harmonic) (Section 3); EUV Off-Plane Mounting (OPM) monochromator preserving the pulse duration (Section 4); Focusing and recombination of the EUV and excitation pulses. The excitation and EUV pulses are focused on the same focal spot on the sample material (Section 5). The sample material is mounted on a five-axis manipulator with liquid helium cooling for Spin-ARPES measurements (Section 6); Ultrafast materials 
engineering stations: MBE and PLD growing the new, ultrafast sample materials. The stations are connected by fast and contamination free UHV transfer tubes to the TR-Spin-ARPES spectrometer (Section 7). Section 8 describes the beamline characterization. Section 9 describes measurements of static and time-resolved ARPES. Finally, Section 10 describes the possible EUV beamline scaling reduction of data acquisition time. This could be achieved by upgrading the laser driver from the $4 \mathrm{~W}$, $1 \mathrm{kHz}$ Ti: Sapphire to the new femtosecond fiber laser with $\sim \mathrm{kW}$ average power, and $\mathrm{MHz}$ repetition rate, resulting in over two orders of magnitude reduction of data acquisition time.

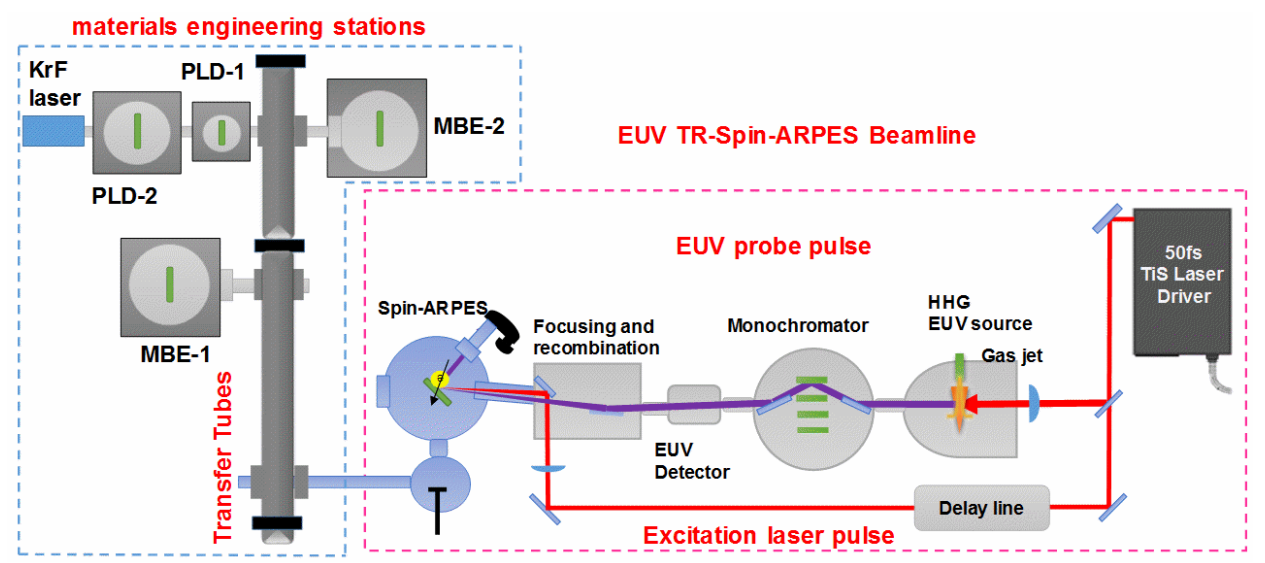

Figure 1. Ultrafast spintronic materials facility has two integral, interconnected parts: the EUV TR-Spin-ARPES Beamline and the materials engineering stations.

(a)

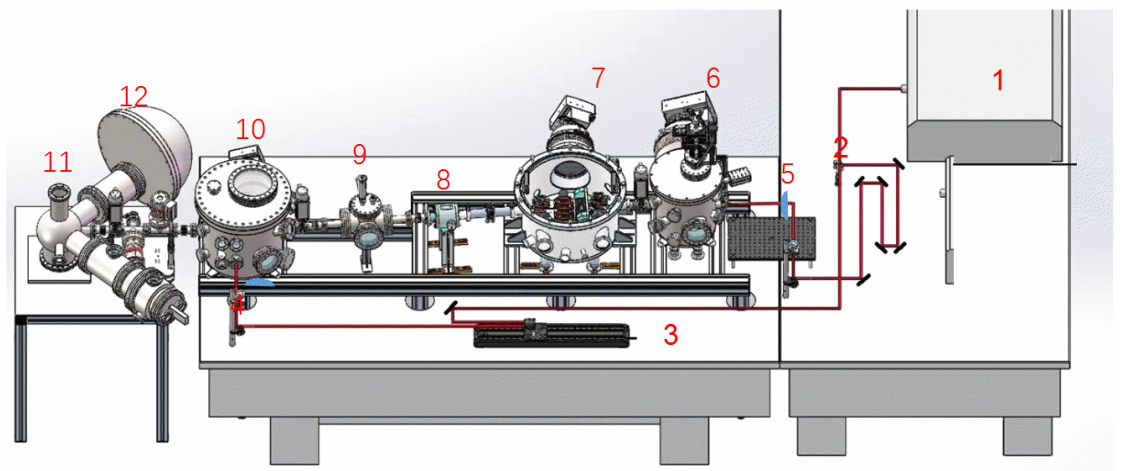

(b)

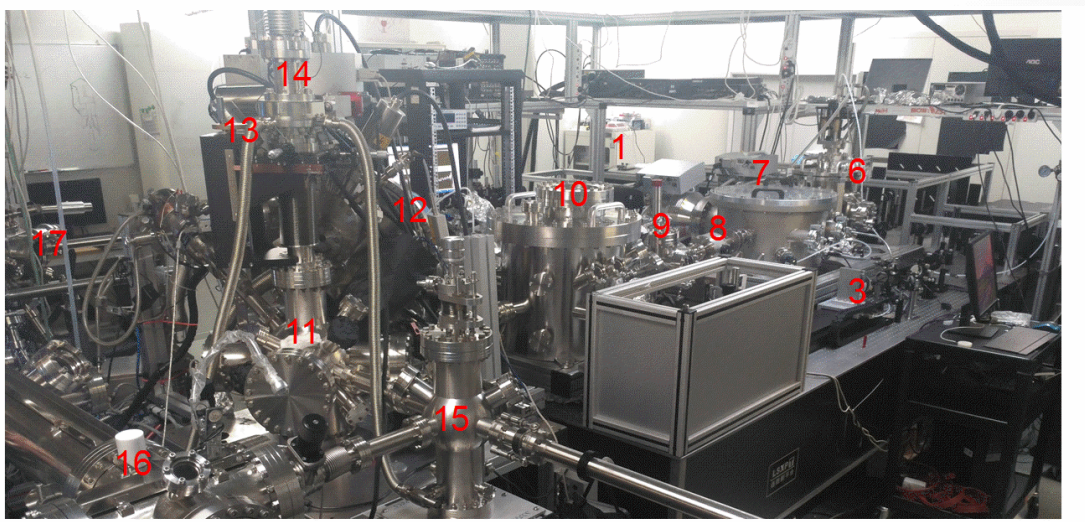

Figure 2. The EUV Beamline: (a) engineering drawing and (b) laboratory picture. (1) TiS laser driver; (2) Laser beam-splitter; (3) Delay line; (4) Focusing lens for excitation pulse); (5) Focusing lens for HHG; (6) HHG EUV source; (7) EUV monochromator; (8) monochromator exit slit; (9) EUV detector chamber; (10) Focusing and recombination chamber; (11) Spin-ARPES spectrometer chamber; (12) Spin-ARPES hemispherical analyzer; (13) Liquid Helium cooling pipes; (14) 4-axis manipulator for sample; (15) Load lock for sample material; (16) Transfer tube bringing sample material from materials engineering stations; (17) MBE-2 materials engineering station. 


\section{Femtosecond Laser-Driver}

The laser-driver is a TiS laser named Libra system from Coherent Inc. ( Santa Clara, CA, USA). The laser emits $\sim 50 \mathrm{fs}$ pulses, centered at $\lambda=800 \mathrm{~nm}$, at $1 \mathrm{kHz}$ repetition rate and with $4 \mathrm{~mJ}$ pulse energy. In the normal operation of the beamline, $2.2 \mathrm{~mJ}$ laser pulses are typically used. They are split in two pulses by an $80 / 20$ beam-splitter: (i) $\approx 1.8 \mathrm{~mJ}$ are focused on the gas jet to generate high harmonics; (ii) the less intense excitation pulse is directed to a delay line and then focused onto the sample in the Spin-ARPES chamber where the pulse energy, after transmission losses, is $\sim 0.2 \mathrm{~mJ}$. The laser has a $10 \mathrm{kHz}$ repetition rate option which will be used in the near future to upgrade the HHG EUV source to a higher repetition rate. At $10 \mathrm{kHz}$, the maximum laser pulse energy is $0.4 \mathrm{~mJ} / \mathrm{pulse}$. In this configuration, tighter laser focusing will be required for HHG.

\section{HHG EUV Source}

The laser-driver is focused by a plano-convex lens into the gas jet to generate the EUV source through the HHG process as shown in Figure 3. The plano-convex lens with a focal length $\mathrm{f}=500 \mathrm{~mm}$ focuses the $1.8 \mathrm{~mJ}$ laser pulse into the gas target with a focal diameter of $\sim 100 \mu \mathrm{m}$ and peak intensity of $\sim 10^{14} \mathrm{~W} / \mathrm{cm}^{2}$. HHG is obtained in Argon or Neon gas jets.

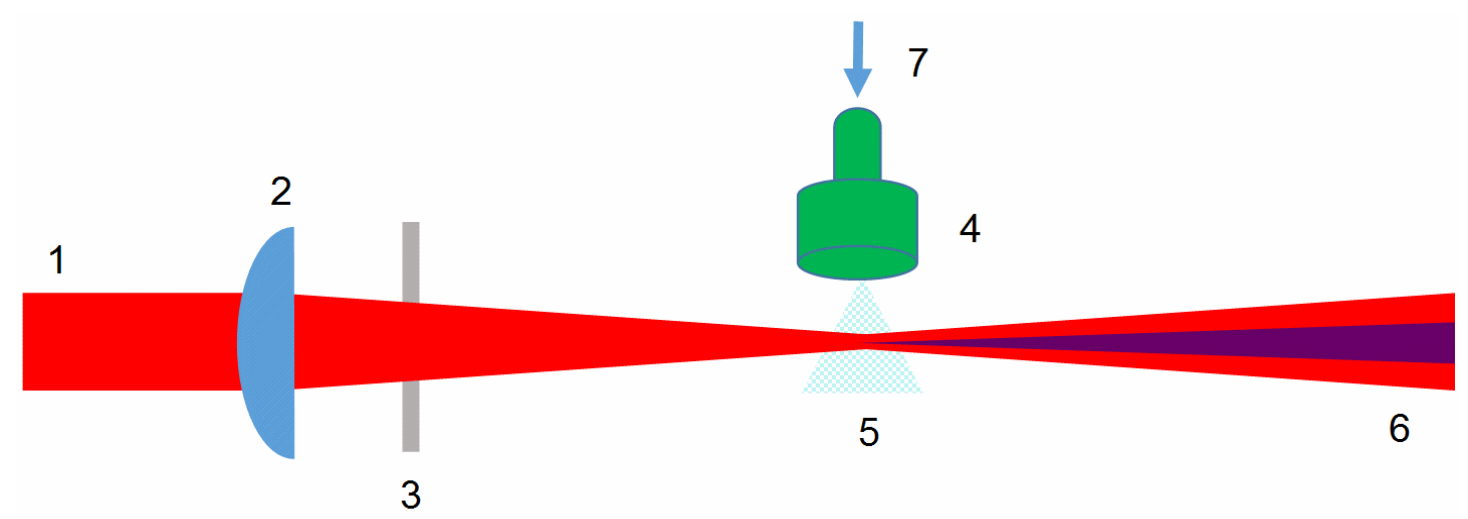

Figure 3. Schematic of the HHG EUV source: (1) input laser pulse; (2) focusing lens; (3) entrance windows with anti-reflective (AR) coating; (4) gas nozzle; (5) HHG EUV source; (6) EUV and laser output to monochromator; and (7) gas input pipe.

The noble gas is injected into the HHG chamber through a pulsed nozzle (Attotech Inc., Staffanstorp, Sweeden) controlled by a pulsed valve with an opening time of $\sim 200 \mu$ s, which is synchronized to the laser's repetition rate. The short opening time of the valve allows a higher gas density to be injected in order to increase the HHG EUV output compared to continuous gas injection-while keeping the overall gas pressure in the HHG chamber to $<10^{-3}$ mbar. The gas backing pressure on the valve is maintained to 2 bar. The position of the gas nozzle relative to the laser focus is optimized by a 3-axis manipulator. To avoid re-absorption of the HHG by the noble gas, the HHG chamber is pumped by a $2200 \mathrm{~L} / \mathrm{s}$ turbo-pump. The base pressure of the chamber is $10^{-3} \mathrm{mbar}$ when gas nozzle is switched on. Differential pumping is used between the HHG chamber and the monochromator, in order to ensure that a pressure of $10^{-6}$ mbar is maintained in the monochromator even when the gas target is turned on.

\section{EUV Pulse-Preserving Monochromator}

The EUV generated from the HHG process has a broad spectrum with equally spaced harmonics. In order to select a single harmonic for time-resolved Spin-ARPES, a pulse preserving EUV monochromator is used after the HHG chamber. The monochromator utilizes gratings in the off-plane mount (OPM). In this design, the EUV incident plane is almost parallel to the grooves of the grating. 
Grating monochromators for EUV ultrafast pulses can be realized using two different designs. The "time-delay compensated" design indicates a monochromator with two grating stages. The first stage performs the monochromatization on an intermediate slit, while the second stage compensates for the pulse front-tilt introduced by the diffraction from the first grating. In this case, the instrument is time-delay compensated and the final temporal response is close to the Fourier limit. Temporal response as short as $5 \mathrm{fs}$ have been recently measured on monochromatized pulses at $42 \mathrm{eV}$, being essentially limited by the intrinsic duration of the generation process [40].

The "time-preserving" design indicates a single-stage grating monochromator in which the operating parameters are chosen so that the pulse temporal broadening (due to the pulse-front tilt) does not alter considerably the pulse duration. In the design here presented, the pulse-front tilt is limited in the few-to-several tens of femtoseconds. The adopted configuration, time preserving in off-plane mount, has the main advantages in the high throughput as well as geometric simplicity [26,27].

The monochromator consists of two toroidal mirrors, a plane grating and a slit with adjustable width, as shown in Figure 4. The first toroidal mirror collimates the beam, while the second one focuses the diffracted beam on the exit slit plane. The monochromator has a set of three interchangeable gratings with different groove density for different spectral ranges and resolutions. Table 1 shows the specifications of toroidal mirrors and three gratings. The width of the slit is adjustable so that the bandwidth of a single harmonic can be controlled to some extent.

(a)

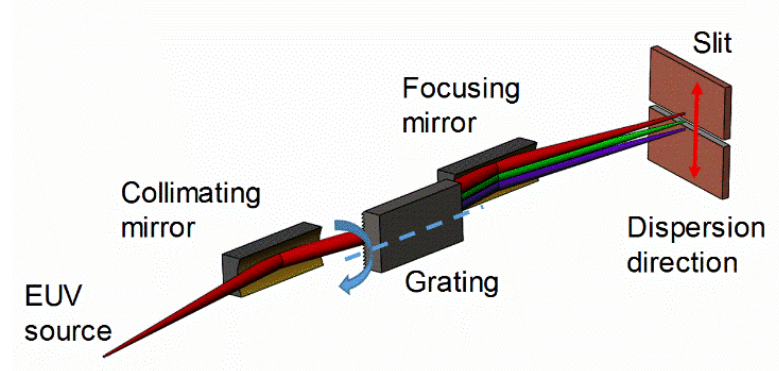

(b)

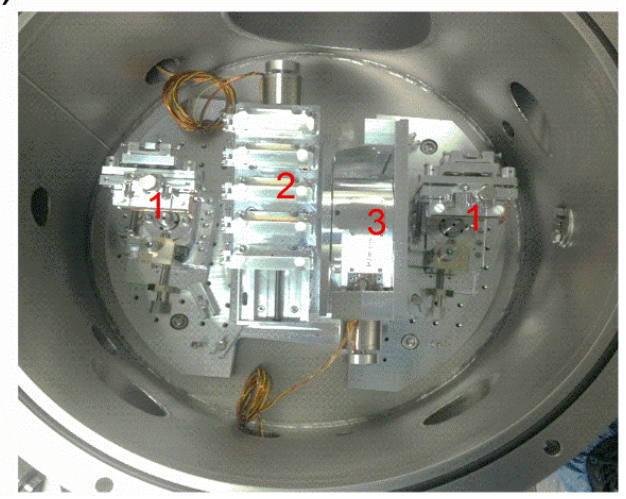

Figure 4. EUV monochromator. (a) off plane mount geometry; (b) laboratory picture: (1) toroidal mirrors; (2) gratings mounted on translation stage; (3) grating rotation stage.

Table 1. Characteristics of the monochromator gratings. The energy resolution has been calculated on a $100-\mu \mathrm{m}$ slit width.

\begin{tabular}{|c|c|c|}
\hline Toroidal Mirrors & $\begin{array}{l}\text { Incident angle } \\
\text { Input/output arms }\end{array}$ & $\begin{array}{l}3^{\circ} \\
500 \mathrm{~mm}\end{array}$ \\
\hline Gratings & Altitude & $3.5^{\circ}$ \\
\hline G1 & $\begin{array}{l}\text { Energy region } \\
\text { Groove density } \\
\text { Bandwidth }(100-\mu \mathrm{m} \text { slit })\end{array}$ & $\begin{array}{l}10.8 \mathrm{eV}-40 \mathrm{eV} \\
150 \mathrm{gr} / \mathrm{mm} \\
\Delta \mathrm{E}=0.4 \mathrm{eV} @ 20 \mathrm{EV}\end{array}$ \\
\hline G2 & $\begin{array}{l}\text { Energy region } \\
\text { Groove density } \\
\text { Bandwidth (100- } \mu \mathrm{m} \text { slit) }\end{array}$ & $\begin{array}{l}10.8 \mathrm{eV}-40 \mathrm{eV} \\
300 \mathrm{gr} / \mathrm{mm} \\
\Delta \mathrm{E}=0.2 \mathrm{eV} @ 20 \mathrm{EV}\end{array}$ \\
\hline G3 & $\begin{array}{l}\text { Energy region } \\
\text { Groove density } \\
\text { Bandwidth }(100-\mu \mathrm{m} \text { slit })\end{array}$ & $\begin{array}{l}30 \mathrm{eV}-100 \mathrm{eV} \\
600 \mathrm{gr} / \mathrm{mm} \\
\Delta \mathrm{E}=0.7 \mathrm{eV} @ 50 \mathrm{EV}\end{array}$ \\
\hline
\end{tabular}

The overall EUV conversion efficiency of the beamline is estimated as $\sim 2 \times 10^{-7} /$ Harmonic, considering a laser to HHG efficiency of $\sim 10^{-6}$ / Harmonic and a beamline overall transmission 
efficiency of $\sim 0.20$. The photon flux at the sample needs anyway to be reduced in order to avoid space charge effects, as discussed in Section 8.5.

\section{Focusing and Recombination of the EUV and Excitation Pulses}

For TR-Spin-ARPES measurements, the IR pump and EUV probe pulses are focused and overlapped spatially and temporally on the sample material, shown in Figure 5 . The EUV monochromatized pulses are focused by a grazing-incidence toroidal mirror used in 1:1 configuration with $1 \mathrm{~m}$ input and output arms, in order to minimize aberration. The diameter of the focused EUV on the sample material could therefore be as small as the EUV source diameter, which is estimated to $<30 \mu \mathrm{m}$. In normal operation, in order to avoid space charge effects at the sample material, the EUV spot size on the sample should be much larger than source size (see Section 8.4). This is achieved by slightly de-focusing the toroidal mirror. The EUV focal spot on the sample material is measured on a Ce: YAG crystal which converts the EUV scattered radiation into visible green light. The excitation laser pulse is also focused on the sample material by a lens with $1.3 \mathrm{~m}$ focal length. The spot-size is adjusted to illuminate an area of about $1 \mathrm{~mm} \times 1.5 \mathrm{~mm}$ in order to provide the optimum excitation fluences on the sample material, typically $1-2 \mathrm{~mJ} / \mathrm{cm}^{2} /$ pulse.

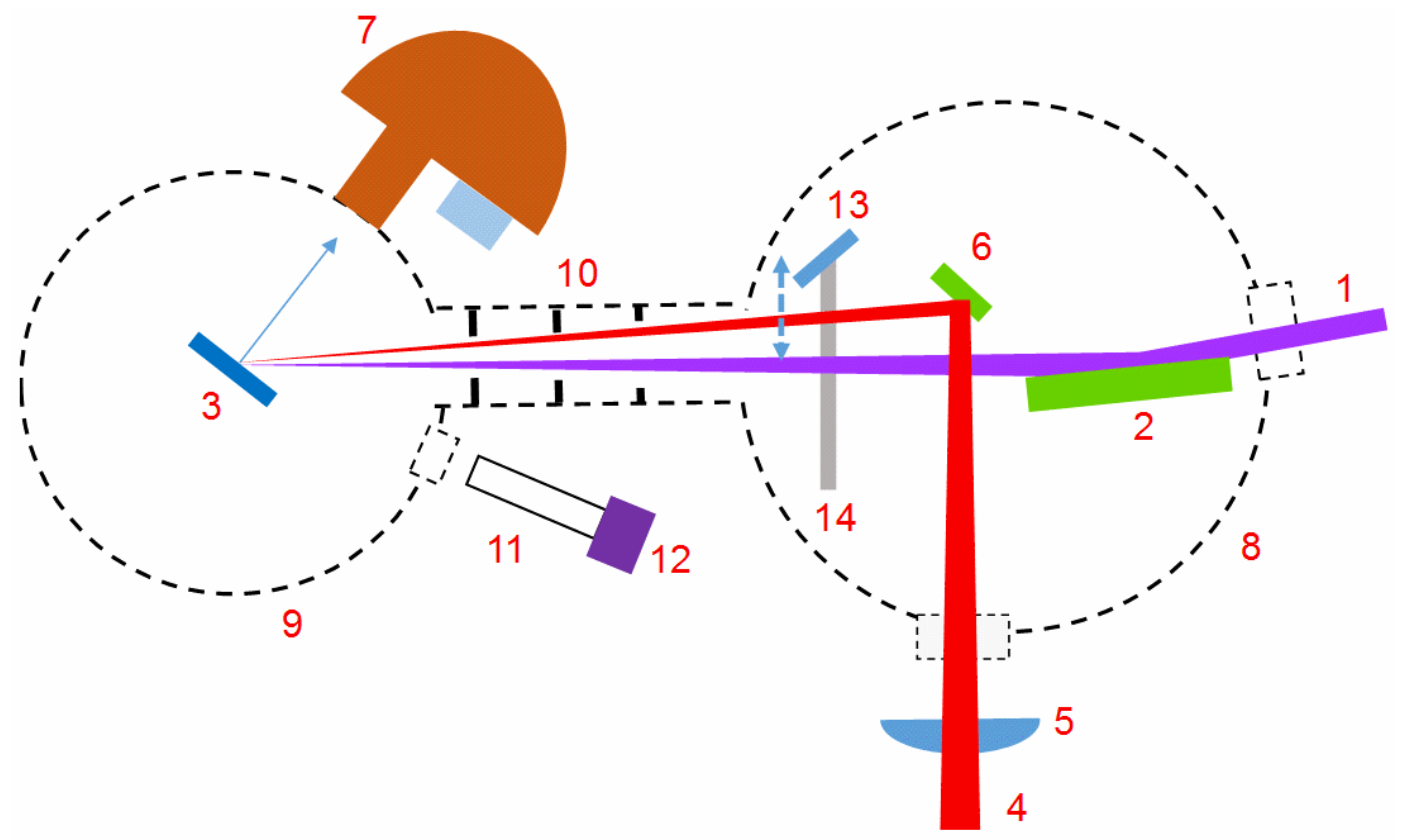

Figure 5. The optical diagram of focusing and recombination chamber: (1) EUV pulse from the monochromator; (2) EUV focusing toroidal mirror on a 5-axis stage; (3) sample materials or CE:YAG crystal; (4) Excitation laser pulse (ELP) from the variable delay line (VDL); (5) AR-coated plano convex lens with a focal length of $\mathrm{f}=1.3 \mathrm{~m}$. (6) The D-shaped re-combination mirror, reflecting ELP; (7) Spin-ARPES detector; (8) Focusing and recombination chamber; (9) Spin-ARPES vacuum chamber; (10) Differential pumping system with three pinholes; (11) Telescope; (12) CCD camera; (13) Plane golden mirror for observing the overlapping of two beams; (14) Linear stage.

The vacuum in the focusing and recombination chamber is maintained at $10^{-8}$ mbar and the ARPES chamber is operated in the $10^{-10}$ mbar vacuum range. The differential pumping between the focusing and recombination chamber and the ARPES chamber is achieved by the three pinholes placed in the pipe between the two chambers.

\section{Spin-ARPES Electron Spectrometers}

The ARPES spectrometer measures: (a) the Kinetic Energy (KE) of the photoelectrons emitted by the sample material when illuminated by EUV photons, and (b) the angles $\theta$ and $\varphi$, representing 
the momentum-vector of photoelectrons emerging from the sample material surface, as shown in Figure 6a. The electron binding energy is calculated by subtracting the measured photoelectron energy from the energy of the EUV photons. The wave-vector $(k)$ is calculated taking the projection of the PE momentum onto the crystal plane. The engineering drawing of the ARPES spectrometer is illustrated in Figure 6b. The PHOIBOS 150 Hemispherical Energy Analyzer can separate photoelectrons with different kinetic energy, supplied by SPECS GmbH (Berlin, Germany). For measurements, two typical detectors are equipped in our Spin-ARPES: one is an imaging CCD for normal electronic band structure, another is a Mott spin detector, which can probe the spin-polarization of electron in the sample materials. The Spin-ARPES chamber is also equipped with a Helium-discharge lamp (UVS300) as UV source. The UVS 300 delivers (always) two emission levels lines: He-I with $21.2 \mathrm{eV}$ and He-II with $40.8 \mathrm{eV}$. Here, we have used He-I as the ARPES source.

(a)

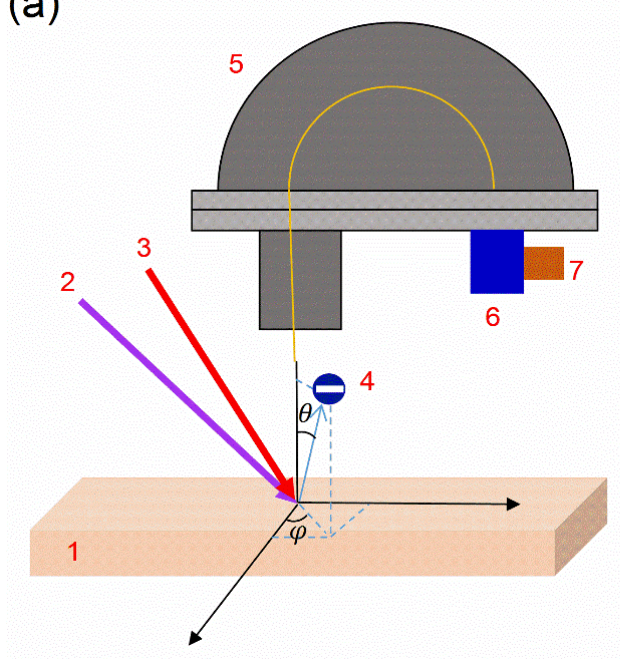

(b)

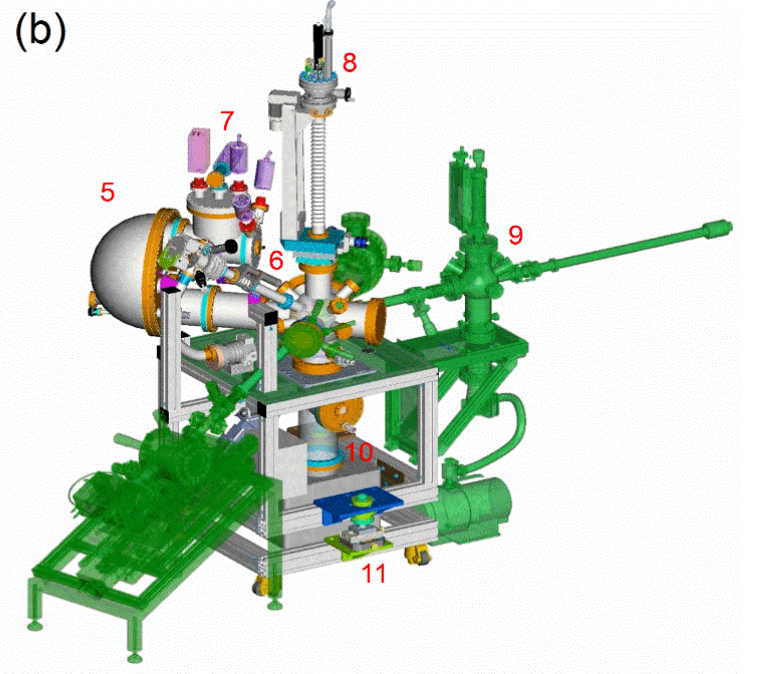

Figure 6. Angle Resolved Photo-emission Spectrometer (ARPES): (a) schematic and (b) engineering drawing. (1) sample material to be analyzed; (2) EUV pulse; (3) excitation laser pulse (ELP); (4) photo-electron (PE); (5) Hemispherical electron analyzer; (6) ARPES detector with multichannel plate (MCP), phosphor screen, telescope and CCD camera; (7) Spin-spectrometer with Mott spin detector; (8) sample material manipulator with five axes and liquid He cooling; (9) load lock for inserting sample material into the ARPES chamber; (10) ultra-high vacuum (UHV) ion pump; (11) XYZ fine mechanical adjustment for positioning the center of the ARPES chamber exactly in the focal-point of the EUV toroidal mirror.

\section{Ultrafast Materials Engineering Stations}

The Ultrafast Spintronic Materials Laboratory integrates the ultrafast materials engineering stations with the EUV Beamline. The new sample materials are engineered in two types of deposition chambers for stacks of single atomic or molecular layers: the Molecular Beam Epitaxy (MBE) stations and the Pulsed Laser Deposition (PLD) stations (Figure 1). The new, ultrafast properties of the engineered sample material are analyzed in the TR-Spin-ARPES spectrometer. To avoid contamination the sample material is transferred directly through the UHV Transfer Tube from the deposition stations to the TR-Spin-ARPES chamber. This fast, contamination-free process will make possible not only analysis but also development of new materials.

\subsection{Molecular Beam Epitaxy Stations}

In the molecular beam epitaxy (MBE) stations, the materials to be deposited are placed in the K-cell Source, heated and evaporated at a time forming molecular beams propagating in UHV towards the substrate. Single atomic or molecular layers of the desired material can be deposited on the substrate. The desired new material is engineered by depositing different atomic layers on the substrate according 
to new designs. The metal-MBE station is equipped with several metal sources like: $\mathrm{Fe}, \mathrm{Co}, \mathrm{Al}, \mathrm{Mg}$ and $\mathrm{Cr}$. The vacuum is maintained at $3 \times 10^{-10} \mathrm{mbar}$. In this station one can grow thin films of magnetic materials for example: $\mathrm{Fe}, \mathrm{Co}_{2} \mathrm{FeAl}, \mathrm{MgO}$, FeOx, etc. [41]. Reflection High Energy Electron Diffraction (RHEED) and longitudinal Magneto-Optical Kerr Effect (MOKE) are setup to monitor the growth process and the magnetic properties of samples in real time. The second MBE station is equipped to deposit semimetals and topological insulators [42,43].

\subsection{Pulsed Laser Deposition Stations}

The PLD stations are used to fabricate high-quality thin films on substrates. A $248 \mathrm{~nm} \mathrm{KrF}$ excimer laser pulse beam ablates the target material. The target molecules acquire kinetic energy and deposit on the atomically flat substrate surface. The laboratory has two PLD stations with a maximum vacuum of $10^{-8}$ mbar: one station is used to fabricate complex oxide thin films such as $\mathrm{LaMnO}_{3}, \mathrm{La}_{x} \mathrm{Sr}_{1-\mathrm{x}} \mathrm{MnO}_{3}$, $\mathrm{LaAlO}_{3}$ and $\mathrm{BaTiO}_{3}[44,45]$ and the second station is used to grow non-oxide [46,47]. An Ozone generator helps optimize the growth process of oxides. During the fabrication of complex oxide films, RHEED is also used to monitor the growth dynamics in order to guarantee the layer-by-layer growth mode.

\section{EUV Beamline Experimental Characterization}

\subsection{HHG EUV Spectra}

The HHG EUV emission spectra from Argon (Ar) and Neon (Ne) were measured by scanning the gratings of the monochromator. The EUV radiation is measured with the absolutely calibrated NIST EUV diode, placed after the slit of the monochromator. The Ar harmonics are generated with 1.8-mJ pulse energy, focused through a lens with focal length $\mathrm{f}=500 \mathrm{~mm}$ on the pulsed gas jet, operating at 1 $\mathrm{kHz}$ repetition rate. Figure 7a shows the photon flux in the HHG spectrum of Ar. The measured flux is above $10^{10} \mathrm{ph} / \mathrm{s}$ for all the harmonics in the range $\mathrm{H} 9-\mathrm{H} 25$, with a maximum emission of $5.5 \times 10^{10}$ $\mathrm{ph} / \mathrm{s}$ at $\mathrm{H} 17(26.4 \mathrm{eV})$, corresponding to $0.23 \mathrm{~nJ} /$ pulse at $1 \mathrm{kHz}$ repetition rate. The flux emitted by the EUV source (before the monochromator) can be estimated to be $\sim 2.5 \times 10^{11} \mathrm{ph} / \mathrm{s}$ at H17, taking into account the monochromator spectral transmission [23]. The Ar spectrum photon energy ranges from $10 \mathrm{eV}$ to $45 \mathrm{eV}$. The $\mathrm{HHG}$ spectrum of $\mathrm{Ne}$ is obtained using higher pulse energy $\sim 3 \mathrm{~mJ} /$ pulse, and shorter focal length $\mathrm{f}=300 \mathrm{~mm}$. Figure $7 \mathrm{~b}$ shows the HHG spectrum of Ne with photon energy extending to $>\mathrm{H} 45(70 \mathrm{eV})$. A maximum photon flux of $3 \times 10^{8} \mathrm{ph} / \mathrm{s}$ is measured at the $\mathrm{H} 29(45 \mathrm{eV})$. The EUV flux measurements show long-term stability over at least 48-h operation, allowing long TR-Spin-ARPES data acquisition runs.

(a)

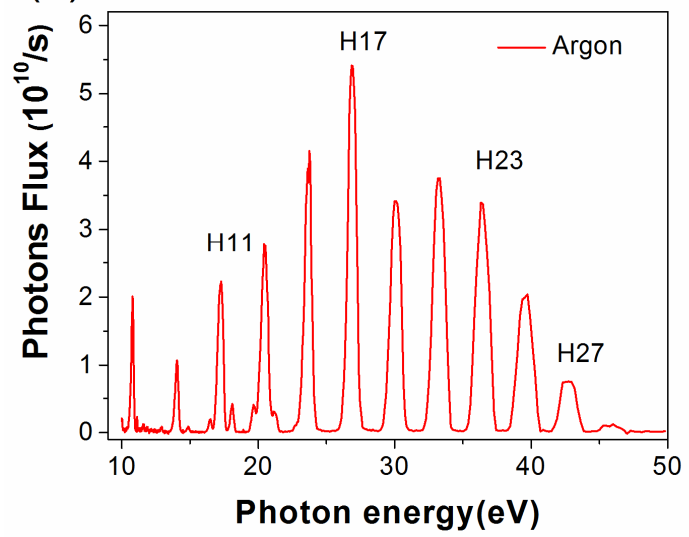

(b)

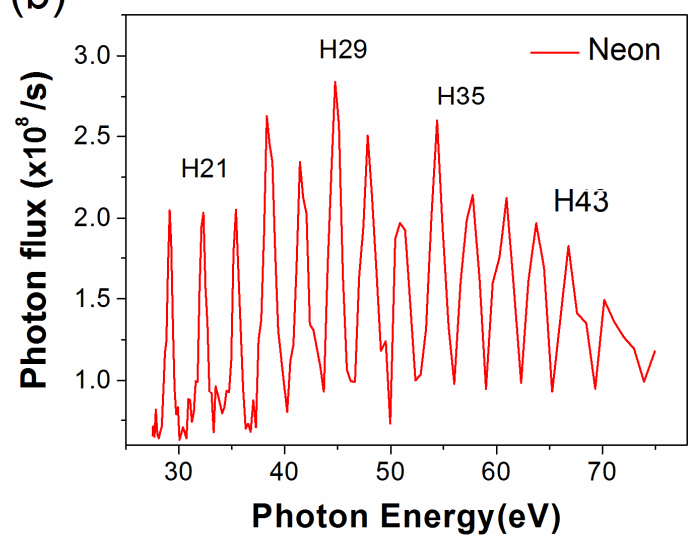

Figure 7. HHG spectra of (a) Ar and (b) Ne, generated with the fundamental TiS laser $\lambda=800 \mathrm{~nm}$ $(1.55 \mathrm{eV})$. The absolute photons flux is measured after the monochromator slit. 
The HHG EUV spectra of Ar and Ne were also produced with 400-nm driving laser pulses [47]. The 1.2-mJ $400 \mathrm{~nm}$ pulses are obtained through second harmonic generation of fundamental laser and then focused on the gas jet with a $\mathrm{f}=300 \mathrm{~mm}$ lens. Figure 8a shows the HHG spectrum in $\mathrm{Ar}$, which has a maximum photon flux of $1.2 \times 10^{11} \mathrm{ph} / \mathrm{s}$ at $\mathrm{H} 7(21.7 \mathrm{eV})$. Figure $8 \mathrm{~b}$ shows the HHG spectrum in $\mathrm{Ne}$, with a maximum photon flux of $5.5 \times 10^{9} \mathrm{ph} / \mathrm{s}$ at $\mathrm{H} 15(46.5 \mathrm{eV})$. The EUV source excited by 400-nm laser can emit higher photon flux, due to the wavelength-scaling effect, and also provide higher energy resolution, which is useful for TR-Spin-ARPES measurements. The spacing between harmonics is increased (Figure 8). Ref. [48] takes advantage of this larger spectral spacing to replace the monochromator with an $\mathrm{Al}$ filter which transmits mainly $\mathrm{H} 7(21.7 \mathrm{eV})$ with bandwidth of $\sim 150 \mathrm{meV}$.

(a)

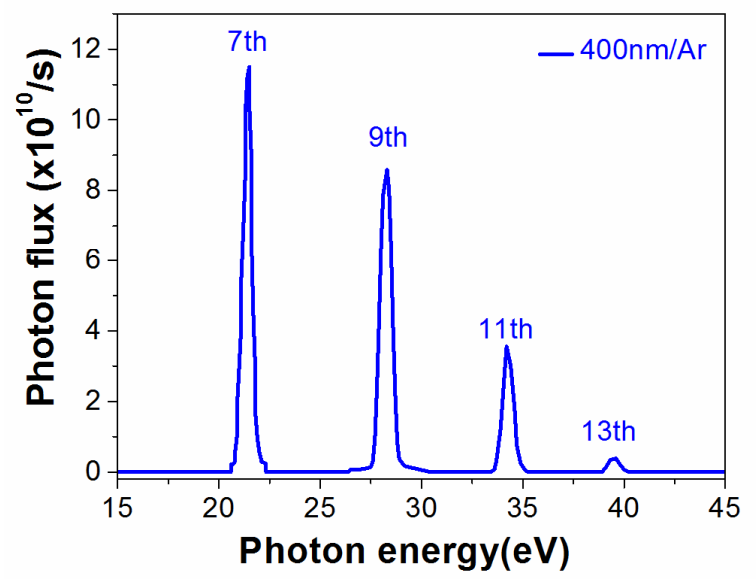

(b)

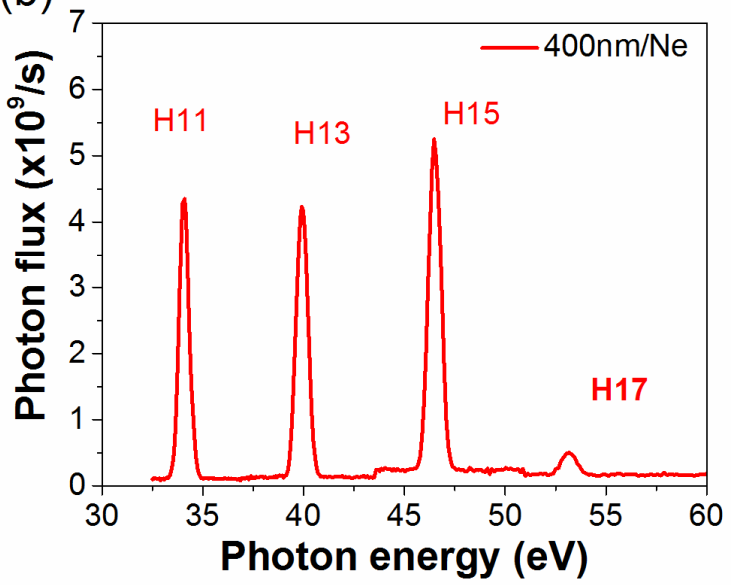

Figure 8. HHG spectra generated by the SHG laser pulse $(400 \mathrm{~nm})$. (a) Ar spectrum measured with grating G2 (300 gr/mm); and (b) Ne spectrum measured with grating G3 (600 gr/mm). The absolute photon flux is measured after the monochromator slit.

\subsection{EUV Spectral Bandwidth}

Photo-electron spectroscopy requires a narrow spectral bandwidth of the EUV radiation. The spectral profile at the output of the monochromator depends on the size of the HHG source, on the monochromator instrumental response (i.e., the bandwidth transmitted through the slit) and on the intrinsic bandwidth of the harmonics. If the intrinsic bandwidth of the harmonics is lower than the bandwidth transmitted through the monochromator, the energy resolution is limited by the source itself. In this case, the monochromator is acting as a broadband filter that is used to select a single harmonic and filter out all the adjacent harmonics. On the other side, if the transmitted bandwidth is lower than the bandwidth of the harmonics, the monochromator, as well as filtering out all the adjacent harmonics, is also selecting a spectral portion of the single harmonic.

The actual bandwidth at the output of the monochromator has been estimated by measuring the HHG spectral profile for different widths of the slit. The widths of the spectral profiles measured for H15 and H21 using G3 are shown in Figure 9. The measured values for H15 and H21 are fitted assuming, respectively, $100-\mu \mathrm{m}$ and $80-\mu \mathrm{m}$ source size and $200-\mathrm{meV}$ and $180-\mathrm{meV}$ source bandwidth, the dashed lines in Figure 9. The size of the source that is found by the fitting procedure $(80-100 \mu \mathrm{m})$ is well in agreement with the measurements of the EUV spot size done on the ARPES chamber using the CE:YAG crystal. Similarly, the source bandwidth (180-200 meV) is in agreement with the resolution measured from the static ARPES spectra, which is $\sim 190 \mathrm{meV}$ after space-charge minimization. Therefore, the actual spectral resolution of the beamline is limited by the intrinsic bandwidth of the source. Indeed, the monochromator acts as a tunable filter with high efficiency in the whole spectral bandwidth of operation. 


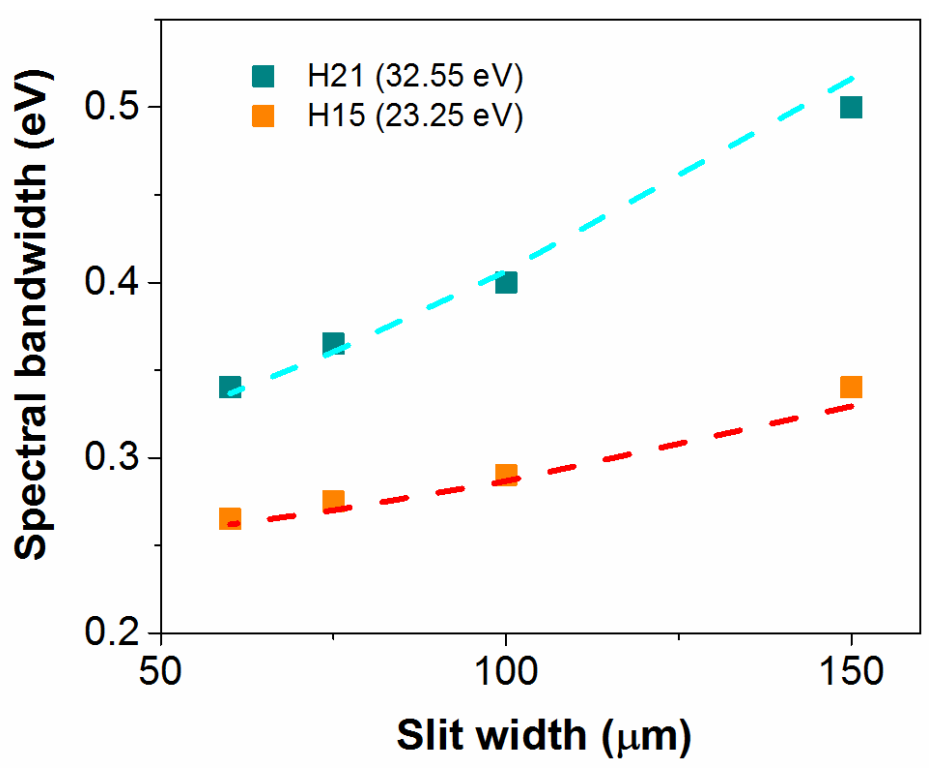

Figure 9. HHG EUV spectral bandwidth measurements as a function of monochromator slit width, grating G3. The dashed lines are simulation results.

\subsection{Pulse Front-Tilt Induced by Gratings: Effect on EUV Pulse Duration}

The grating gives intrinsically a wavefront tilt due to the diffraction phenomenon. In fact, each illuminated groove gives a delay of one wavelength at first diffraction order; therefore, the total delay results as $\Delta \mathrm{OP}=\mathrm{N} \lambda$, where $\mathrm{N}$ is the number of illuminated grooves and $\lambda$ is the EUV wavelength. Therefore, the wavefront tilt is directly proportional to the grating illuminated area. Once the beam divergence is measured, the resulting wavefront tilt is

$$
\mathrm{WFT}=\mathrm{DIV} \times \mathrm{L} \times \sigma \times \lambda \times \mathrm{c}^{-1},
$$

where DIV is the EUV beam divergence, $\mathrm{L}$ is the distance between the HHG source and the first toroidal mirror, $\sigma$ is the grating groove density, $\lambda$ is the wavelength and $c$ is the speed of light in vacuum. The beam divergence has been measured through the knife-edge test, resulting in $3.1 \mathrm{mrad}$ for $\mathrm{H} 15(23.5 \mathrm{eV})$ and $2.6 \mathrm{mrad}$ for $\mathrm{H} 21(32.55 \mathrm{eV})$. The corresponding wavefront tilt is reported for the three gratings in Table 2. In the same table, the bandwidth on a $100-\mu \mathrm{m}$ slit is also reported, in order to show the typical trade-off in the design of a monochromator for ultrafast pulses: the narrower the bandwidth, the longer the time response, and vice versa.

Table 2. EUV wavefront tilt given by the three gratings for $\mathrm{H} 15$ and $\mathrm{H} 21$.

\begin{tabular}{ccccc}
\hline & \multicolumn{2}{c}{ H15 (23.50 eV) } & \multicolumn{2}{c}{ H21 (32.55 eV) } \\
\hline & $\begin{array}{c}\text { Wavefront Tilt at Half } \\
\text { Maximum (fs) }\end{array}$ & $\begin{array}{c}\text { Bandwidth on } \\
\mathbf{1 0 0} \boldsymbol{\mu} \text { m Slit (meV) }\end{array}$ & $\begin{array}{c}\text { Wavefront Tilt at Half } \\
\text { Maximum (fs) }\end{array}$ & $\begin{array}{c}\text { BANDWIDTH on } \\
\mathbf{1 0 0} \boldsymbol{\mu} \text { m Slit (meV) }\end{array}$ \\
\hline G150 & 20 & 580 & 12 & 1140 \\
G300 & 40 & 290 & 25 & 570 \\
G600 & 80 & 145 & 50 & 285 \\
\hline
\end{tabular}

\subsection{EUV Focused Spot Size on the Sample Material}

The EUV beam emerging from the monochromator is focused by a toroidal mirror on the surface of sample material, placed in the center of the ARPES chamber. We choose a large EUV focal spot size in order to avoid a space charge effect on the samples. To measure the size of the EUV focal spot, a CE:YAG crystal is placed into the ARPES chamber in the exact position of the sample. Figure 10a shows the EUV focal spot of the H15 $(23.25 \mathrm{eV})$ on the CE:YAG crystal. The $x$-axis and $y$-axis dimensions of 
the EUV spot are fitted with a Gaussian function and the corresponding Full-Width-Half- Maximum (FWHM) are $\mathrm{D}_{\mathrm{x}-\mathrm{FWHM}}=170 \mu \mathrm{m}$ and $\mathrm{D}_{\mathrm{y}-\mathrm{FWHM}}=120 \mu \mathrm{m}$, respectively, as shown in Figure 10b. The focal spot is elongated along the $x$-axis because the EUV beam is incident at $45^{\circ}$ on the crystal.
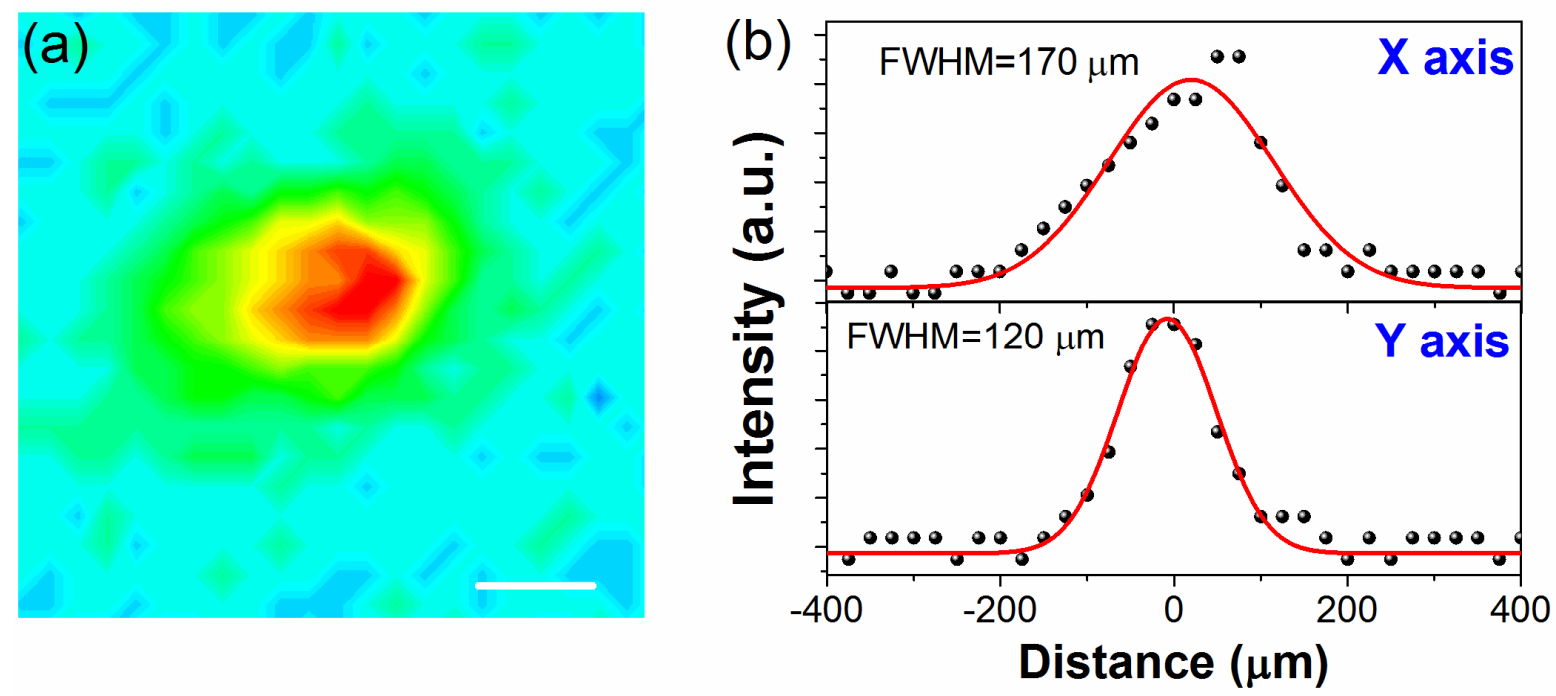

Figure 10. EUV spot size measurement (a) CCD image of the fluorescence of the CE:YAG crystal, irradiated with EUV beam; scale bar is $100 \mu \mathrm{m}$; (b) the measured focal spot profiles $\mathrm{D}_{\mathrm{x}-\mathrm{FWHM}} \sim 170 \mu \mathrm{m}$ and $\mathrm{D}_{\mathrm{y}-\mathrm{FWHM}} \sim 120 \mu \mathrm{m}$.

\subsection{Space Charge Effect at the Sample Material}

While the EUV photon flux of $>10^{10} \mathrm{ph} / \mathrm{s}$ after the monochromator is comparable to the flux of synchrotron radiation source beamlines, the number of photons per pulse is much higher because of the lower pulse repetition rate of the laser, compared with the synchrotron. The large photons density incident on the sample surface during the ultrashort EUV pulse can lead to the space charge effect, which can distort photoelectron emission. A single-crystal copper $\mathrm{Cu}(111)$ as the sample was inserted into the ARPES chamber and its Fermi-edge photoemission spectra were measured in order to measure the influence of space charge on the ARPES spectra and characterize the energy resolution of the EUV beamline. The $\mathrm{H} 13(20.15 \mathrm{eV})$ is chosen with grating G2 as photon source and the slit width is $\sim 100 \mu \mathrm{m}$. The photon flux was varied from $5.7 \times 10^{9}$ to $6 \times 10^{8} \mathrm{ph} / \mathrm{s}$. Figure 11 shows the effect of space charge on the ARPES measurements of the copper crystal. In Figure 11a, we observe space charge broadening and shifting of the Fermi-edge for higher photon fluxes. The space charge effect is minimized for $\mathrm{F}_{\mathrm{ph}} \sim 3.6 \times 10^{9} \mathrm{ph} / \mathrm{s}$, which corresponds to a pulse photon density of $180 \mathrm{ph} / \mu \mathrm{m} / \mathrm{pulse}$ in the EUV focal spot with dimensions of $170 \mu \mathrm{m} \times 120 \mu \mathrm{m}$. The Fermi-edge spectrum was fitted with Fermi-Dirac function, convolved with a $190 \mathrm{meV}$-width Gaussian function (black line in Figure 11b). The good fitting shows that energy resolution of the EUV beamline is $\sim 190 \mathrm{meV}$ for H13, when space charge effects are minimized. This spectral resolution is in agreement with the design resolution of grating G2.

In order to minimize the space charge effects, we need to reduce the EUV pulse photon density on the sample to $<180 \mathrm{ph} / \mu \mathrm{m}^{2} /$ pulse. This pulse photon density is in good agreement with the space-charge measurements in Ref. [23] and is typical for TR-ARPES measurements reported in Refs. [28,29] for example. The reduction in photon density is obtained by increasing the EUV focal spot area at the sample, to a maximum of $170 \mu \mathrm{m} \times 120 \mu \mathrm{m}$, and/or reducing the photon flux at the sample.

The EUV spot size of an average diameter of $\sim 150 \mu \mathrm{m}$ has a negligible contribution to the angular resolution and hence to the momentum measurement. On SRS, the EUV spot size is around $50 \mu \mathrm{m}$ and the effect of the $150 \mu \mathrm{m}$ in the TR-ARPES is negligible due to the low energy resolution $(190 \mathrm{meV})$. Essentially in TR-ARPES, the momentum resolution is dominated by the energy resolution of the photon beam. 
(a)

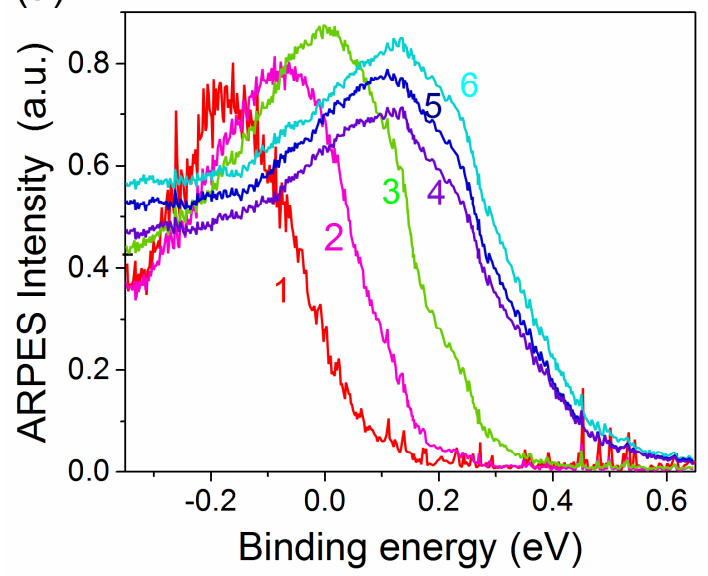

(b)

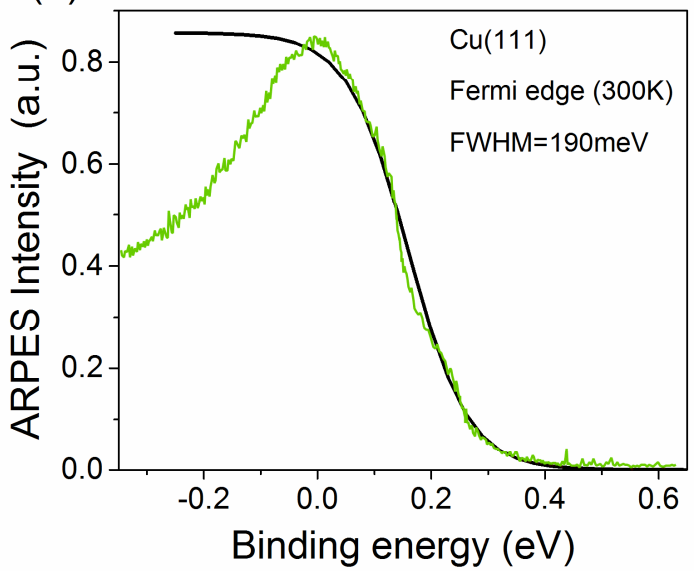

Figure 11. ARPES spectra of the Fermi edge of $\mathrm{Cu}(111)$ as a function of incident EUV flux on the sample material. EUV photon energy H13 $(20.15 \mathrm{eV})$; monochromator grating G2 and slit width = 100 $\mu \mathrm{m}$. (a) spectra measured with the following EUV photon fluxes (ph/s): (1) $6 \times 10^{8}$; (2) $1.5 \times 10^{9}$; (3) $3.6 \times 10^{9}$; (4) $4.8 \times 10^{9}$; (5) $5.28 \times 10^{9}$; (6) $5.68 \times 10^{9}$; (b) the Fermi edge measured with photon flux $\sim 3.6 \times 10^{9} \mathrm{ph} / \mathrm{s}$, corresponding to a pulse photon density $\sim 180\left(\mathrm{ph} / \mu \mathrm{m}^{2}\right)$. The black fitting curve has a $\mathrm{FWHM}=190 \mathrm{meV}$.

In angular resolving lens modes, the emission angle distribution is imaged on the detector. Here, the finite spot size of the photon beam on the sample contributes to the angular resolution and therefore to the momentum resolution. Theoretically, all electrons with the same escape angle excited from within the photon beam spot should be focused into an infinitely small spot on the detector. In reality, this spot on the detector will have a certain lateral spread $\mathrm{M}$, which could also be viewed as a magnification. The contribution of the photon beam spot size $S$ to the angular resolution A may be described by

$$
\mathrm{A}=\frac{\mathrm{M}}{\mathrm{D}} \times \mathrm{S},
$$

where $\mathrm{D}$ is the angular dispersion of the lens mode. For example, the Wide Angle Mode (WAM) of the PHOIBOS 150 analyzer has an angular acceptance of $\pm 15^{\circ}$ with $\mathrm{D}=0.75 \mathrm{~mm} /{ }^{\circ}$. The values for $\mathrm{M}$ depend on the lens mode and the retardation ratio $\mathrm{R}=$ kinetic energy/pass energy. For the WAM mode, $\mathrm{M}$ is typically of the value of 0.8 . and the specification for the angular resolution is $0.5^{\circ}$. Therefore, a photon beam spot size $\mathrm{S}$ of $0.15 \mathrm{~mm}$ will result in a broadening $\mathrm{A}$ of $0.15^{\circ}$. This is much smaller than the specified angular resolution of $0.5^{\circ}$ for this particular lens mode.

\section{TR-ARPES Spectra Measurements}

\subsection{Static ARPES Spectra of $\mathrm{Cu}$ (111)}

Except for the investigation of space charge effect, static ARPES spectra of single-crystal copper were also measured at room temperature, using the EUV beamline with photon energy H13 $(20.15 \mathrm{eV})$. The EUV photon flux was adjusted to $\sim 6 \times 10^{8} \mathrm{ph} / \mathrm{s}$ and the EUV spot area at the sample was $170 \mu \mathrm{m}$ $\times 120 \mu \mathrm{m}$. Grating G2 was used and the silt width was Ws $=100 \mu \mathrm{m}$. The exposure time for acquiring data was $\sim 20 \mathrm{~min}$. Figure 12a shows a snapshot of the Shockley surface state of $\mathrm{Cu}(111)$, along $\Gamma-\mathrm{K}$ direction in the surface Brillouin zone. For comparison, Figure 12b shows the similar ARPES image obtained with radiation from an He-I discharge lamp $(21.22 \mathrm{eV})$. 
(a)

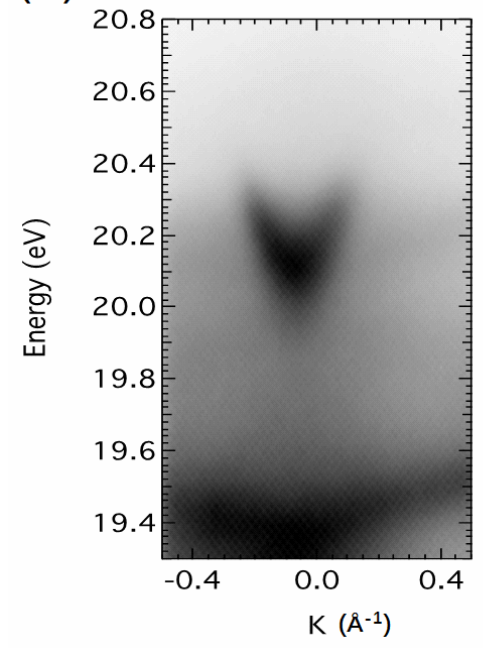

(b)

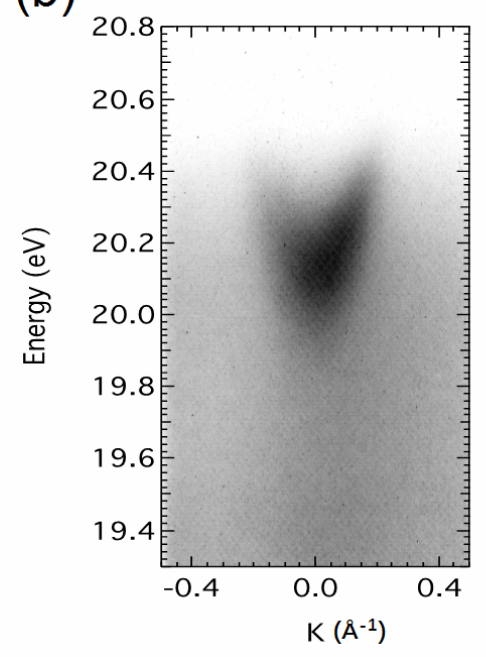

Figure 12. ARPES spectra of $\mathrm{Cu}$ (111) band structure, along the $\Gamma K$-direction in the surface Brillouin zone. (a) HHG EUV beam with H13 $\left(h v_{13}=20.15 \mathrm{eV}\right)$; and $(\mathbf{b}) \mathrm{He}-\mathrm{I}$ lamp $\left(h v_{\mathrm{He}}=21.22 \mathrm{eV}\right)$. Vertical axis: PE energy $(\mathrm{eV})$ and horizontal axis: electron $\mathrm{K}$-vectors $\left(\AA^{-1}\right)$.

\subsection{TR ARPES Spectra of p-type GaAs (100)}

TR-ARPES spectra of p-type GaAs (100) valence band were measured with time delay steps $\sim 67$ fs from time-zero to $1000 \mathrm{fs}$ and larger steps up to a delay of $30 \mathrm{ps}$ (Figure 13). The 50 -fs excitation laser pulse $(1.55 \mathrm{eV})$ irradiates the sample material with a fluence of $2 \mathrm{~mJ} / \mathrm{cm}^{2}$. The femtosecond EUV pulse with photon energy $\sim 20.15 \mathrm{eV}$ (H13) probes the excited sample material with photon flux $\sim 10^{9} \mathrm{ph} / \mathrm{s}$ (photon density $\sim 50 \mathrm{ph} / \mu \mathrm{m}^{2} /$ pulse).

Figure 13a shows the ARPES spectra of the valence band of GaAs (100) at time zero (left image) and at time delay $+650 \mathrm{fs}$ (right image) after the excitation laser pulse. To further distinguish the influence of excitation pulse on the ARPES spectra, two typical electron Energy Distribution Curves (EDC) are plotted in Figure 13b, for two time delays: $-500 \mathrm{fs}$ (before excitation) and $+5 \mathrm{ps}$ (after excitation). It is clearly observed that the peak position of EDC is shifted to the higher kinetic energy side when the EUV probe pulse arrives at the sample after the excitation. We attributed this up-shifting phenomenon to the Surface Photo-Voltaic (SPV) effect. In general, the downward band bending on the p-type semiconductor can trap photo-excited electrons at the surface and drive photo-excited holes into the bulk. Such electron-hole separation could produce additional electric flied to transiently shift the band structure up in energy $[49,50]$.

The shift of EDC peaks in valence band (called by SPV shift), induced by the excitation laser pulse, can be calculated from the temporal evolution of EDC:

$$
\text { SPV shift }=\text { KEEDC-PEAK }(T D L)-K E_{\text {EDC-PEAK }}(T Z),
$$

where KE $E_{\text {EDC-PEAK }}(\mathrm{TDL})$ is the kinetic energy (KE) of photoelectrons at the peak of EDC for a given time-delay (TDL); and KE $E_{\text {EDC-PEAK }}(\mathrm{TZ}$ ) is the value at the time zero (TZ). Figure 13c,d show the SPV shift of GaAs's valence band as a function of time delay between excitation laser pulse and EUV probe pulse. Within ultrafast rise-time $\sim 650 \mathrm{fs}$, the SPV shift reaches a maximum plateau of $\sim 80 \mathrm{meV}$, lasting $>30 \mathrm{ps}$. The SPV error bars are obtained by averaging out the measured data over 50 cycles. The errors are minimized by measuring $\mathrm{KE}_{\mathrm{EDC}-\mathrm{PEAK}}$ at the peak of the EDC. The temporal step error bars are not shown because they are much smaller than the delay step $\sim 67 \mathrm{fs}$. Similar SPV measurements of photo-excited electron dynamics in p-type GaAs $(100)$ have previously been reported $[49,50]$. 
(a)

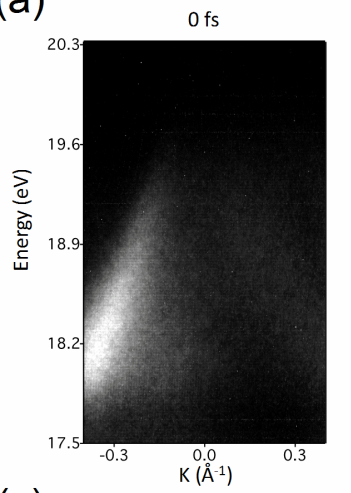

(c)

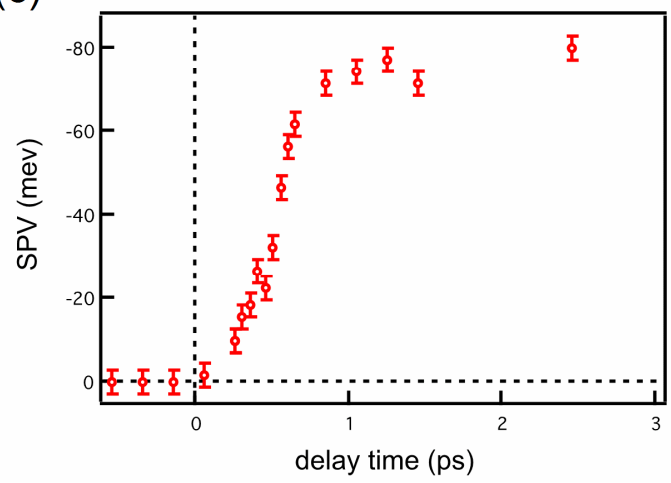

(b)

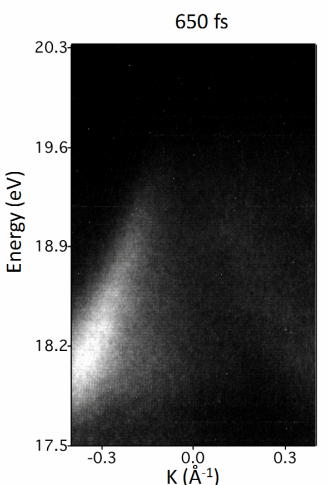

(d)
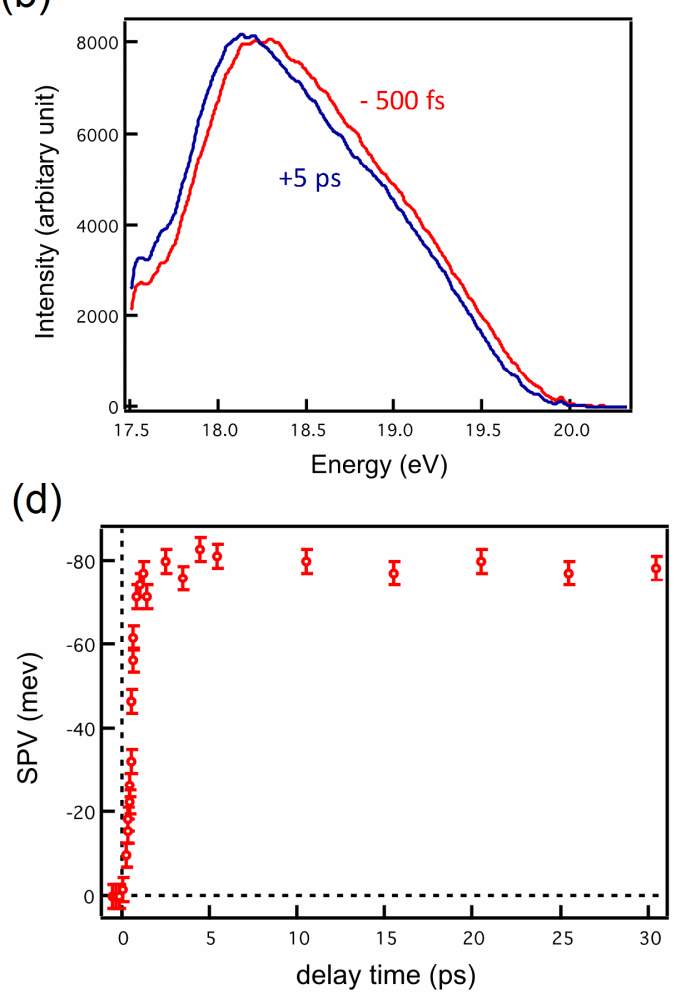

Figure 13. TR-ARPES measurements of the ultrafast surface photovoltaic (SPV) effect in p-GaAs (100). (a) TR ARPES spectra of the valence band near the $\Gamma$ point at $0 \mathrm{fs}$ and $650 \mathrm{fs}$ delay after the excitation pulse. Vertical axis: photoelectron (PE) energy (eV); Horizontal axis: PE momenta $\mathrm{k}\left(\AA^{-1}\right)$; (b) typical energy distribution curves (EDC) of valence band measured before the excitation pulse ( $-500 \mathrm{fs})$ and after the excitation pulse ( $+5 \mathrm{ps}$ ); (c) temporal evolution of SPV effect with $650 \mathrm{fs}$ rise-time. Delay steps are $67 \mathrm{fs}$; (d) SPV effect over showing a plateau lasting $>30$ ps.

\section{EUV Beamline Scaling to a $1 \mathrm{MHz}$ Repetition Rate}

The $1 \mathrm{KHz}$ repetition rate of the present laser-driver leads to quite long data acquisition time (tens of hours), which could not only reduce the efficiency of measurements, but also increases the experimental error. In addition, it could limit the investigation of extremely sensitive but novel materials. The repetition rate and the useful photon flux at the sample material (inside TR-Spin-ARPES spectrometer) could be scaled by a factor of up to $\sim 1000 \times$. This could be achieved by upgrading the present laser driver to the $\mathrm{MHz}$ repetition rate and $\sim \mathrm{kW}$ average power, femtosecond fiber-lasers. Such laser is now becoming available $[51,52]$ and has already been used to produce high EUV fluxes by HHG [52]. The $1 \mathrm{MHz}$ repetition rate, for example, could reduce the data acquisition time from tens of hours at $1 \mathrm{kHz}$ to minutes at $1 \mathrm{MHz}$, for a complete set of TR-ARPES runs.

Figure 14 shows a schematic of such an upgraded EUV beamline system. The $\sim 1.2 \mathrm{~kW}$ average power, $\mathrm{MHz}$ fiber laser output would be $\sim 1.2 \mathrm{~mJ} /$ pulse, $\sim 300 \mathrm{fs}$ pulse duration, $\lambda=1030 \mathrm{~nm}(1.2 \mathrm{eV})$. The laser pulse could be compressed to $<40 \mathrm{fs}$, in a hollow fiber with an output of $\sim 0.7 \mathrm{~mJ} / \mathrm{pulse}$, which is only $\sim 2.6 \times$ smaller than present pulse energy. The upgraded EUV beamline would operate similarly to the present EUV BL. A small fraction of the laser pulse energy would be split to drive the excitation laser pulse, and the main laser pulse energy drives the HHG EUV source.

The large laser average power, $\sim \mathrm{kW}$, will require special heat management to protect the EUV beamline from damage. The laser beam would be transformed into an annular beam, with a small central hole, prior to focusing in the HHG chamber. The HHG source would use a gas jet and cooled gas nozzle. The laser beam would be separated from the EUV beam immediately after HHG. This could be achieved by taking advantage of the laser annular beam and larger divergence compared to the 
EUV beam. The EUV beam passes through the hole of the mirror while the laser power is reflected by the mirror into a cooled beam-dump. Alternatively, the reflected beam could be recycled to run a second HHG beamline. The laser power loss in the HHG process is very small.

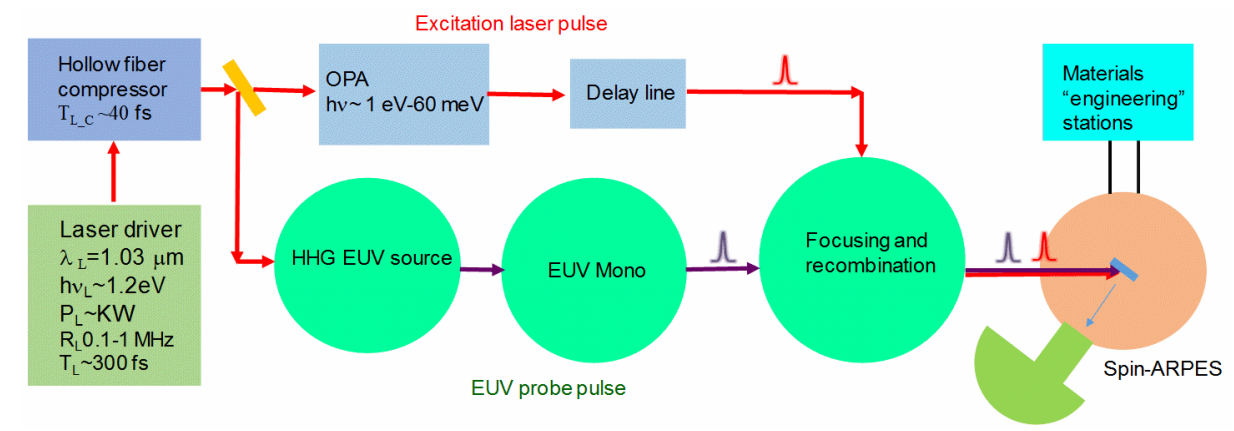

Figure 14. The EUV Beamline scaling to $\sim \mathrm{MHz}, \sim \mathrm{kW}$ class femtosecond fiber laser-driver.

The repetition rate could be increased further, for example to $20 \mathrm{MHz}$ (or even $100 \mathrm{MHz}$ ) by using the same $\mathrm{kW}$-class fibre laser-driver laser with $20 \times($ or $100 \times)$ reduced pulse energy compared to $1 \mathrm{MHz}$ (Active Fibre Systems GmbH, Jena, Germany, Data Sheet). As the Beamline repetition rate is increased above tens of $\mathrm{MHz}$, consideration needs to be given to the effects of heat diffusion and/or material relaxation in the sample. These very high repetition rates may restrict the option of using Mid-Infrared excitation pulse, as proposed in Figure 14 and the paragraph below. An alternative method of achieving these very high EUV beamline repetition rates is using a Yb:fibre frequency comb of $80 \mathrm{~W}$ average power and cavity-enhanced high-harmonic source to obtain repetition of $88 \mathrm{MHz}$ [53] and $60 \mathrm{MHz}$ [54]. The beamlines provide EUV pulses of $\sim 100 \mathrm{fs}$ [53] and $400 \mathrm{fs}$ [54] with $65 \mathrm{meV}$ and $<25 \mathrm{meV}$ spectral resolution, respectively, with sub-100 $\mu \mathrm{m}$ EUV spot on the sample material. This interesting new scheme would require major modifications to the present beamline as well as a high finesse cavity with cavity length locked to the seed.

An optional Beamline geometry could provide femtosecond mid-IR excitation laser as in Ref. [20], instead of the fundamental laser photons $(\mathrm{h} v=1.2 \mathrm{eV})$. This could be achieved by using the excitation laser pulse to drive an optical parametric amplifier (OPA) as shown in Figure 14. The OPA would generate femtosecond pulses of mid-IR (hv 1 eV-60 meV or $\lambda \sim 1.2 \mu \mathrm{m}-20 \mu \mathrm{m}$ ) which are well suited to excite only neighboring energy levels to the one of interest $[20,26]$ for TR-Spin-ARPES spectroscopy. This option could be implemented with the fiber laser-driver operating at $\sim 100 \mathrm{kHz}$ and generating $\sim 7 \mathrm{~mJ} /$ pulse in $<40 \mathrm{fs}$ pulses. The laser pulse energy would be divided into: $\sim 6 \mathrm{~mJ} /$ pulse driving the OPA, and $\sim 1 \mathrm{~mJ} /$ pulse driving the HHG EUV source. This option could provide a $\sim 100 \times$ increase in the useful EUV photon/flux at the sample material. Such a beamline will also reduce the TR-Spin-ARPES DAQ time from tens of hours at $1 \mathrm{kHz}$ repetition rate to tens of minutes at $100 \mathrm{kHz}$.

\section{Conclusions}

A new femtosecond EUV beamline was developed for ultrafast materials research and development. The beamline is an integral part of the "Ultrafast Spintronic Materials Facility" at Nanjing University. The facility also comprises materials engineering stations by molecular beam epitaxy (MBE), sputtering and pulsed laser deposition (PLD), all in the same experimental area. This leads to a fast turn-around time from the production of a new sample material to its being analyzed in the TR-Spin-ARPES station. Therefore, the facility will provide not only fast "analysis" but also "development" of new ultrafast materials for the electronics industry.

The EUV beamline is composed of the: 50 -fs laser driver; excitation laser pulse beamline; HHG EUV source, pulse-preserving EUV monochromator; focusing and recombination of the EUV and excitation laser pulse; and Spin-ARPES spectrometer end-station. The EUV beamline was fully characterized and optimized for TR-Spin-ARPES spectroscopy. The HHG EUV spectra from 
$10 \mathrm{eV}-75 \mathrm{eV}$ were measured after the monochromator slit. A maximum flux of $\sim 5.5 \times 10^{10} \mathrm{ph} / \mathrm{s}$ is measured at the $\mathrm{H} 17(26.4 \mathrm{eV})$ using Argon gas and $3 \times 10^{8} \mathrm{ph} / \mathrm{s}$ is measured at the $\mathrm{H} 29(45 \mathrm{eV})$ using Neon gas. The EUV flux is increased when the SHG of the TiS laser $(400 \mathrm{~nm})$ is used to drive the HHG, due to the wavelength-scaling effect. The spectral bandwidth and wavefront tilt of the EUV beamline can be measured and in good agreement of calculation. The EUV monochromator can enable the narrow bandwidth of the EUV beamline and also preserve its pulse duration. The space charge effect on static ARPES spectra was measured as a function of EUV pulse photon density at the sample material. The space charge effect is minimized when the photon density is below $200 \mathrm{ph} / \mu \mathrm{m}^{2} / \mathrm{pulse}$. This is obtained by controlling the EUV flux and focal spot-size on sample material.

Time resolved ARPES measurements of the of $\mathrm{p}$-GaAs (100) were performed over 30 ps temporal delay range, with fine temporal steps of $67 \mathrm{fs}$ for the first $1000 \mathrm{fs}$ delay between the excitation laser pulse and the EUV pulse. TR-ARPES spectra measured the ultrafast surface photovoltaic effect with a $650 \mathrm{fs}$ rise-time of the valence band electronic structure.

The TR-Spin-ARPES data acquisition time could be reduced from tens of hours to minutes by upgrading the femtosecond TiS, $\mathrm{kHz}, 4 \mathrm{~W}$ laser driver to the new, femtosecond, $\sim \mathrm{MHz}$ repetition rate and $\sim \mathrm{kW}$ average power fiber-lasers.

Author Contributions: I.C.E.T., L.H., L.P., Y.L., Z.N. (Zhonghui Nie) and Y.X. conceived and designed the experiments; Z.N. (Zhonghui Nie), Y.L., J.T. and Z.N. (Zhiqiang Ni) performed the construction of the EUV beamline; F.F., P.M. and N.F. designed and constructed the EUV monochromator; Z.N. (Zhonghui Nie), Y.L., J.T., Z.N. (Zhiqiang Ni), F.F., and P.M. performed the EUV beamline characterization and optimization; X.Z., H.X., X.R., J.W., Q.L., C.L., T.K., and C.C. performed the measurement of ARPES and TR-APRES; Y.C. and X.W. performed the materials engineering in the growth stations; Y.Z., W.L., F.W., Y.S., and R.Z. provided helpful advice during the construction of the whole facility. All authors participated in writing the manuscript.

Funding: This research was funded by the National Natural Science Foundation of China (No. 61427812) and the National Key Research and Development Program of China (No. 2016YFA0300803, No. 2017YFA0206304).

Acknowledgments: Z.N. (Zhonghui Nie), I.C.E.T., Y.L., X.Z., L.H., J.T., Z.N. (Zhiqiang Ni), H.X., Y.C., X.R., X.W., F.W., Y.S., R.Z., and Y.X. acknowledge the support from the National Natural Science Foundation of China (No. 11774160, 11574137, 61474061, 61674079, 61805116, 61822403, 11874203 and 11504159), the National Basic Research Program of China (No. 2014CB921101), the Jiangsu Shuangchuang Program, the Natural Science Foundation of Jiangsu Province of China (No. BK20140054, BK20180056), the UK EPSRC (EP/S010246/1) and Program for a high-level Entrepreneurial and Innovative Talent Introduction, Jiangsu Province; L.P., F.F., P.M., and N.F. acknowledge the support from the Extreme Light Infrastructures (ELI) funds of the Italian Ministry for Education, University and Research; C.L. and Q.L. acknowledge the support from the National Natural Science Foundation of China (NSFC) (No. 11504159), NSFC Guangdong (No. 2016A030313650), the Technology and Innovation Commission of Shenzhen Municipality (No. ZDSYS20170303165926217, No. JCYJ20170412152620376, No. JCYJ20150630145302240), and the Guangdong Innovative and Entrepreneurial Research Team Program (Grant No. 2016ZT06D348).

Conflicts of Interest: The authors declare no conflict of interest.

\section{References}

1. Damascelli, A.; Hussain, Z.; Shen, Z.X. Angle-resolved photoemission studies of the cuprate superconductors. Rev. Mod. Phys. 2003, 75, 473-541. [CrossRef]

2. Koralek, J.D.; Douglas, J.F.; Plumb, N.C.; Griffith, J.D.; Cundiff, S.T.; Kapteyn, H.C.; Murnane, M.M.; Dessau, D.S. Experimental setup for low-energy laser-based angle resolved photoemission spectroscopy. Rev. Sci. Instrum. 2007, 78, 053905. [CrossRef] [PubMed]

3. Koralek, J.D.; Douglas, J.F.; Plumb, N.C.; Sun, Z.; Fedorov, A.V.; Murnane, M.M.; Kapteyn, H.C.; Cundiff, S.T.; Aiura, Y.; Oka, K.; et al. Laser based angle-resolved photoemission, the sudden approximation, and quasiparticle-like spectral peaks in Bi2Sr2CaCu2O(8+delta). Phys. Rev. Lett. 2006, 96, 017005. [CrossRef]

4. Strickland, D.; Mourou, G. Compression of amplified chirped optical pulses. Opt. Commun. 1985, 55, 447-449. [CrossRef]

5. Liu, G.; Wang, G.; Zhu, Y.; Zhang, H.; Zhang, G.; Wang, X.; Zhou, Y.; Zhang, W.; Liu, H.; Zhao, L.; et al. Development of a vacuum ultraviolet laser-based angle-resolved photoemission system with a superhigh energy resolution better than $1 \mathrm{meV}$. Rev. Sci. Instrum. 2008, 79, 023105. [CrossRef] [PubMed] 
6. Meng, J.; Liu, G.; Zhang, W.; Zhao, L.; Liu, H.; Jia, X.; Mu, D.; Liu, S.; Dong, X.; Zhang, J.; et al. Coexistence of Fermi arcs and Fermi pockets in a high-T(c) copper oxide superconductor. Nature 2009, 462, 335-338. [CrossRef] [PubMed]

7. He, S.; He, J.; Zhang, W.; Zhao, L.; Liu, D.; Liu, X.; Mou, D.; Ou, Y.B.; Wang, Q.Y.; Li, Z.; et al. Phase diagram and electronic indication of high-temperature superconductivity at $65 \mathrm{~K}$ in single-layer FeSe films. Nat. Mater. 2013, 12, 605-610. [CrossRef] [PubMed]

8. Berntsen, M.H.; Gotberg, O.; Tjernberg, O. An experimental setup for high resolution $10.5 \mathrm{eV}$ laser-based angle-resolved photoelectron spectroscopy using a time-of-flight electron analyzer. Rev. Sci. Instrum. 2011, 82, 095113. [CrossRef]

9. Ahmadivand, A.; Semmlinger, M.; Dong, L.; Gerislioglu, B.; Nordlander, P.; Halas, N.J. Toroidal Dipole-Enhanced Third Harmonic Generation of Deep Ultraviolet Light using Plasmonic Meta-Atoms. Nano Lett. 2018. [CrossRef]

10. Semmlinger, M.; Tseng, M.L.; Yang, J.; Zhang, M.; Zhang, C.; Tsai, W.Y.; Tsai, D.P.; Nordlander, P.; Halas, N.J. Vacuum Ultraviolet Light-Generating Metasurface. Nano Lett. 2018. [CrossRef]

11. Lisowski, M.; Loukakos, P.A.; Bovensiepen, U.; Stahler, J.; Gahl, C.; Wolf, M. Ultra-fast dynamics of electron thermalization, cooling and transport effects in Ru(001). Appl. Phys. A Mater. Sci. Process. 2004, 78, 165-176. [CrossRef]

12. Schmitt, F.; Kirchmann, P.S.; Bovensiepen, U.; Moore, R.G.; Rettig, L.; Krenz, M.; Chu, J.H.; Ru, N.; Perfetti, L.; Lu, D.H.; et al. Transient electronic structure and melting of a charge density wave in TbTe3. Science 2008, 321, 1649-1652. [CrossRef] [PubMed]

13. Smallwood, C.L.; Jozwiak, C.; Zhang, W.; Lanzara, A. An ultrafast angle-resolved photoemission apparatus for measuring complex materials. Rev. Sci. Instrum. 2012, 83, 123904. [CrossRef] [PubMed]

14. Smallwood, C.L.; Hinton, J.P.; Jozwiak, C.; Zhang, W.; Koralek, J.D.; Eisaki, H.; Lee, D.H.; Orenstein, J.; Lanzara, A. Tracking Cooper pairs in a cuprate superconductor by ultrafast angle-resolved photoemission. Science 2012, 336, 1137-1139. [CrossRef] [PubMed]

15. Graf, J.; Jozwiak, C.; Smallwood, C.L.; Eisaki, H.; Kaindl, R.A.; Lee, D.-H.; Lanzara, A. Nodal quasiparticle meltdown in ultrahigh-resolution pump-probe angle-resolved photoemission. Nat. Phys. 2011, 7, 805-809. [CrossRef]

16. Sobota, J.A.; Yang, S.; Analytis, J.G.; Chen, Y.L.; Fisher, I.R.; Kirchmann, P.S.; Shen, Z.X. Ultrafast optical excitation of a persistent surface-state population in the topological insulator Bi2Se3. Phys. Rev. Lett. 2012, 108, 117403. [CrossRef] [PubMed]

17. Mcpherson, A.; Gibson, G.; Jara, H.; Johann, U.; Luk, T.S.; Mcintyre, I.A.; Boyer, K.; Rhodes, C.K. Studies of Multiphoton Production of Vacuum Ultraviolet-Radiation in the Rare-Gases. J. Opt. Soc. Am. B 1987, 4, 595-601. [CrossRef]

18. Ferray, M.; Lhuillier, A.; Li, X.F.; Lompre, L.A.; Mainfray, G.; Manus, C. Multiple-Harmonic Conversion of 1064-Nm Radiation in Rare-Gases. J. Opt. Soc. Am. B 1988, 21, L31-L35. [CrossRef]

19. Li, X.F.; Lhuillier, A.; Ferray, M.; Lompre, L.A.; Mainfray, G. Multiple-Harmonic Generation in Rare-Gases at High Laser Intensity. Phys. Rev. A 1989, 39, 5751-5761. [CrossRef]

20. Corkum, P.B. Plasma perspective on strong field multiphoton ionization. Phys. Rev. Lett. 1993, 71, $1994-1997$. [CrossRef]

21. Jaegle, P. Coherent Sources of XUV Radiation; Springer: Berlin, Germany, 2006; pp. 106-416.

22. Park, I.-Y.; Kim, S.; Choi, J.; Lee, D.-H.; Kim, Y.-J.; Kling, M.F.; Stockman, M.I.; Kim, S.-W. Plasmonic generation of ultrashort extreme-ultraviolet light pulses. Nat. Photonics 2011, 5, 677-681. [CrossRef]

23. Turcu, I.C.E.; Springate, E.; Froud, C.A.; Cacho, C.M.; Collier, J.L.; Bryan, W.A.; Nemeth, G.R.A.J.; Marangos, J.P.; Tisch, J.W.G.; et al. Ultrafast science and development at the Artemis facility. Proc. SPIE 2009, 7469, 746902. [CrossRef]

24. Froud, C.A.; Langley, A.J.; Springate, E.; Turcu, I.C.E.; Wolff, D.S.; Cavalleri, A.; Underwood, J.; Dhesi, S.S.; Bonora, S.; Frassetto, F.; et al. Artemis: A sub 10-fs XUV source for ultrafast time resolved science. In Central Laser Facility Annual Report 2006-2007; Rutherford Appleton Laboratory: Swindon, UK, 2006-2007; RAL Report No. RAL-TR-2007-025; pp. 173-175. ISBN 9780955661617.

25. Artemis. Available online: https://www.clf.stfc.ac.uk/Pages/Artemis.aspx (accessed on 12 December 2018). 
26. Frassetto, F.; Cacho, C.; Froud, C.A.; Turcu, I.C.; Villoresi, P.; Bryan, W.A.; Springate, E.; Poletto, L. Single-grating monochromator for extreme-ultraviolet ultrashort pulses. Opt. Express 2011, 19, 19169-19181. [CrossRef]

27. Poletto, L.; Frassetto, F. Time-preserving grating monochromators for ultrafast extreme-ultraviolet pulses. Appl. Opt. 2010, 49, 5465-5473. [CrossRef] [PubMed]

28. Petersen, J.C.; Kaiser, S.; Dean, N.; Simoncig, A.; Liu, H.Y.; Cavalieri, A.L.; Cacho, C.; Turcu, I.C.; Springate, E.; Frassetto, F.; et al. Clocking the melting transition of charge and lattice order in 1T-TaS2 with ultrafast extreme-ultraviolet angle-resolved photoemission spectroscopy. Phys. Rev. Lett. 2011, 107, 177402. [CrossRef] [PubMed]

29. Gierz, I.; Petersen, J.C.; Mitrano, M.; Cacho, C.; Turcu, I.C.; Springate, E.; Stohr, A.; Kohler, A.; Starke, U.; Cavalleri, A. Snapshots of non-equilibrium Dirac carrier distributions in graphene. Nat. Mater. 2013, 12, 1119-1124. [CrossRef] [PubMed]

30. Cacho, C.; Crepaldi, A.; Battiato, M.; Braun, J.; Cilento, F.; Zacchigna, M.; Richter, M.C.; Heckmann, O.; Springate, E.; Liu, Y.; et al. Momentum-resolved spin dynamics of bulk and surface excited States in the topological insulator $\mathrm{Bi}_{2} \mathrm{Se}_{3}$. Phys. Rev. Lett. 2015, 114, 097401. [CrossRef]

31. Mathias, S.; Miaja-Avila, L.; Murnane, M.M.; Kapteyn, H.; Aeschlimann, M.; Bauer, M. Angle-resolved photoemission spectroscopy with a femtosecond high harmonic light source using a two-dimensional imaging electron analyzer. Rev. Sci. Instrum. 2007, 78, 083105. [CrossRef]

32. Dakovski, G.L.; Li, Y.; Durakiewicz, T.; Rodriguez, G. Tunable ultrafast extreme ultraviolet source for timeand angle-resolved photoemission spectroscopy. Rev. Sci. Instrum. 2010, 81, 073108. [CrossRef]

33. Wernet, P.; Gaudin, J.; Godehusen, K.; Schwarzkopf, O.; Eberhardt, W. Femtosecond time-resolved photoelectron spectroscopy with a vacuum-ultraviolet photon source based on laser high-order harmonic generation. Rev. Sci. Instrum. 2011, 82, 063114. [CrossRef]

34. Frietsch, B.; Carley, R.; Dobrich, K.; Gahl, C.; Teichmann, M.; Schwarzkopf, O.; Wernet, P.; Weinelt, M. A high-order harmonic generation apparatus for time- and angle-resolved photoelectron spectroscopy. Rev. Sci. Instrum. 2013, 84, 075106. [CrossRef]

35. Grazioli, C.; Callegari, C.; Ciavardini, A.; Coreno, M.; Frassetto, F.; Gauthier, D.; Golob, D.; Ivanov, R.; Kivimaki, A.; Mahieu, B.; et al. CITIUS: An infrared-extreme ultraviolet light source for fundamental and applied ultrafast science. Rev. Sci. Instrum. 2014, 85, 023104. [CrossRef] [PubMed]

36. Jordan, I.; Huppert, M.; Brown, M.A.; van Bokhoven, J.A.; Worner, H.J. Photoelectron spectrometer for attosecond spectroscopy of liquids and gases. Rev. Sci. Instrum. 2015, 86, 123905. [CrossRef] [PubMed]

37. Ojeda, J.; Arrell, C.A.; Grilj, J.; Frassetto, F.; Mewes, L.; Zhang, H.; van Mourik, F.; Poletto, L.; Chergui, M. Harmonium: A pulse preserving source of monochromatic extreme ultraviolet (30-110 eV) radiation for ultrafast photoelectron spectroscopy of liquids. Struct. Dyn. 2016, 3, 023602. [CrossRef] [PubMed]

38. Rohde, G.; Hendel, A.; Stange, A.; Hanff, K.; Oloff, L.P.; Yang, L.X.; Rossnagel, K.; Bauer, M. Time-resolved ARPES with sub-15 fs temporal and near Fourier-limited spectral resolution. Rev. Sci. Instrum. 2016, 87, 103102. [CrossRef] [PubMed]

39. Schmidt, J.; Guggenmos, A.; Chew, S.H.; Gliserin, A.; Hogner, M.; Kling, M.F.; Zou, J.; Spath, C.; Kleineberg, U. Development of a $10 \mathrm{kHz}$ high harmonic source up to $140 \mathrm{eV}$ photon energy for ultrafast time-, angle-, and phase-resolved photoelectron emission spectroscopy on solid targets. Rev. Sci. Instrum. 2017, 88, 083105. [CrossRef] [PubMed]

40. Lucchini, M.; Lucarelli, G.D.; Murari, M.; Trabattoni, A.; Fabris, N.; Frassetto, F.; De Silvestri, S.; Poletto, L.; Nisoli, M. Few-femtosecond extreme-ultraviolet pulses fully reconstructed by ptychographic technique. Opt. Express 2018, 26, 6771-6784. [CrossRef] [PubMed]

41. Zhang, X.; Xu, H.; Lai, B.; Lu, Q.; Lu, X.; Chen, Y.; Niu, W.; Gu, C.; Liu, W.; Wang, X.; et al. Direct observation of high spin polarization in Co2FeAl thin films. Sci. Rep. 2018, 8, 8074. [CrossRef]

42. Liu, W.Q.; He, L.; Zhou, Y.; Murata, K.; Onbasli, M.C.; Ross, C.A.; Jiang, Y.; Wang, Y.; Xu, Y.B.; Zhang, R.; et al. Evidence for ferromagnetic coupling at the doped topological insulator/ferrimagnetic insulator interface. AIP Adv. 2016, 6, 055813. [CrossRef]

43. Liu, W.; West, D.; He, L.; Xu, Y.; Liu, J.; Wang, K.; Wang, Y.; van der Laan, G.; Zhang, R.; Zhang, S.; et al. Atomic-Scale Magnetism of Cr-Doped Bi2Se3 Thin Film Topological Insulators. ACS Nano 2015, 9, 10237-10243. [CrossRef] 
44. Niu, W.; Gao, M.; Wang, X.; Song, F.; Du, J.; Wang, X.; Xu, Y.; Zhang, R. Evidence of weak localization in quantum interference effects observed in epitaxial La0.7Sr0.3MnO3 ultrathin films. Sci. Rep. 2016, 6, 26081. [CrossRef] [PubMed]

45. Niu, W.; Liu, W.Q.; Gu, M.; Chen, Y.D.; Zhang, X.Q.; Zhang, M.H.; Chen, Y.Q.; Wang, J.; Du, J.; Song, F.Q.; et al. Direct Demonstration of the Emergent Magnetism Resulting from the Multivalence $\mathrm{Mn}$ in a $\mathrm{LaMnO}_{3}$ Epitaxial Thin Film System. Adv. Electron. Mater. 2018, 4. [CrossRef]

46. Gao, M.; Zhang, M.H.; Niu, W.; Chen, Y.Q.; Gu, M.; Wang, H.Y.; Song, F.Q.; Wang, P.; Yan, S.C.; Wang, F.Q.; et al. Tuning the transport behavior of centimeter-scale WTe2 ultrathin films fabricated by pulsed laser deposition. Appl. Phys. Lett. 2017, 111. [CrossRef]

47. Gao, W.B.; Huang, L.; Xu, J.L.; Chen, Y.Q.; Zhu, C.H.; Nie, Z.H.; Li, Y.; Wang, X.F.; Xie, Z.D.; Zhu, S.N.; et al. Broadband photocarrier dynamics and nonlinear absorption of PLD-grown WTe2 semimetal films. Appl. Phys. Lett. 2018, 112. [CrossRef]

48. Eich, S.; Stange, A.; Carr, A.V.; Urbancic, J.; Popmintchev, T.; Wiesenmayer, M.; Jansen, K.; Ruffing, A.; Jakobs, S.; Rohwer, T.; et al. Time- and angle-resolved photoemission spectroscopy with optimized high-harmonic pulses using frequency-doubled Ti:Sapphire lasers. J. Electron. Spectrosc. Relat. Phenom. 2014, 195, 231-236. [CrossRef]

49. Tanaka, S.; More, S.D.; Murakami, J.; Itoh, M.; Fujii, Y.; Kamada, M. Surface photovoltage effects on p-GaAs (100) from core-level photoelectron spectroscopy using synchrotron radiation and a laser. Phys. Rev. B 2001, 64. [CrossRef]

50. Azuma, J.; Tokudomi, S.; Takahashi, K.; Kamada, M. Dynamics of photo-excited carriers on GaAs(100) surface studied by ultrafast time-resolved photoemission. Phys. Status Solidi C 2009, 6, 307-310. [CrossRef]

51. Muller, M.; Kienel, M.; Klenke, A.; Gottschall, T.; Shestaev, E.; Plotner, M.; Limpert, J.; Tunnermann, A. 1 kW $1 \mathrm{~mJ}$ eight-channel ultrafast fiber laser. Opt. Lett. 2016, 41, 3439-3442. [CrossRef]

52. Hadrich, S.; Klenke, A.; Rothhardt, J.; Krebs, M.; Hoffmann, A.; Pronin, O.; Pervak, V.; Limpert, J.; Tunermann, A. High photon flux table-top coherent extreme-ultraviolet source. Nat. Photonics 2014, 8 , 779-783. [CrossRef]

53. Corder, C.; Zhao, P.; Bakalis, J.; Li, X.; Kershis, M.D.; Muraca, A.R.; White, M.G.; Allison, T.K. Ultrafast extreme ultraviolet photoemission without space charge. Struct. Dyn. 2018, 5, 054301. [CrossRef]

54. Mills, A.K.; Zhdanovich, S.; Boschini, F.; Na, M.X.; Schneider, M.; Dosanjh, P.; Wong, D.; Levy, G.; Damascelli, A.; Jones, D.J. Time-resolved Femtosecond Photoemission Spectroscopy using a $60-\mathrm{MHz}$ Enhancement Cavity XUV Source. In Proceedings of the 2017 Conference on Lasers and Electro-Optics (CLEO), San Jose, CA, USA, 14-19 May 2017. 